Article

\title{
Strong Convergence of a System of Generalized Mixed Equilibrium Problem, Split Variational Inclusion Problem and Fixed Point Problem in Banach Spaces
}

\author{
Mujahid Abbas ${ }^{1,2}$, Yusuf Ibrahim ${ }^{3}$, Abdul Rahim Khan ${ }^{4}$ and Manuel de la Sen ${ }^{5, *(1)}$ \\ 1 Department of Mathematics, Government College University, Katchery Road, Lahore 54000, Pakistan; \\ abbas.mujahid@gmail.com \\ 2 Department of Mathematics and Applied Mathematics University of Pretoria, Pretoria 0002, South Africa \\ 3 Department of Mathematics, Sa'adatu Rimi College of Education, Kumbotso Kano, P.M.B. 3218 Kano, \\ Nigeria; danustazz@gmail.com \\ 4 Department of Mathematics and Statistics, King Fahad University of Petroleum and Minerals, \\ Dhahran 31261, Saudi Arabia; arahim@kfupm.edu.sa \\ 5 Institute of Research and Development of Processes, University of The Basque Country, \\ Campus of Leioa (Bizkaia), 48080 Leioa, Spain \\ * Correspondence: manuel.delasen@ehu.eus
}

Received: 2 April 2019; Accepted: 21 May 2019; Published: 27 May 2019

check for updates

\begin{abstract}
The purpose of this paper is to introduce a new algorithm to approximate a common solution for a system of generalized mixed equilibrium problems, split variational inclusion problems of a countable family of multivalued maximal monotone operators, and fixed-point problems of a countable family of left Bregman, strongly asymptotically non-expansive mappings in uniformly convex and uniformly smooth Banach spaces. A strong convergence theorem for the above problems are established. As an application, we solve a generalized mixed equilibrium problem, split Hammerstein integral equations, and a fixed-point problem, and provide a numerical example to support better findings of our result.
\end{abstract}

Keywords: split variational inclusion problem; generalized mixed equilibrium problem; fixed point problem; maximal monotone operator; left Bregman asymptotically nonexpansive mapping; uniformly convex and uniformly smooth Banach space

\section{Introduction and Preliminaries}

Let $E$ be a real normed space with dual $E^{*}$. A map $B: E \rightarrow E^{*}$ is called:

(i) monotone if, for each $x, y \in E,\langle\eta-v, x-y\rangle \geq 0, \forall \eta \in B x, v \in B y$, where $\langle\cdot, \cdot\rangle$ denotes duality pairing,

(ii) $\epsilon$-inverse strongly monotone if there exists $\epsilon>0$, such that $\langle B x-B y, x-y\rangle \geq \epsilon\|B x-B y\|^{2}$,

(iii) maximal monotone if $B$ is monotone and the graph of $B$ is not properly contained in the graph of any other monotone operator. We note that $B$ is maximal monotone if, and only if it is monotone, and $R(J+t B)=E^{*}$ for all $t>0, J$ is the normalized duality map on $E$ and $R(J+t B)$ is the range of $(J+t B)$ (cf. [1]). 
Let $H_{1}$ and $H_{2}$ be Hilbert spaces. For the maximal monotone operators $B_{1}: H_{1} \rightarrow 2^{H_{1}}$ and $B_{2}: H_{2} \rightarrow 2^{H_{2}}$, Moudafi [2] introduced the following split monotone variational inclusion:

$$
\begin{aligned}
& \text { find } x^{*} \in H_{1} \text { such that } 0 \in f\left(x^{*}\right)+B_{1}\left(x^{*}\right), \\
& y^{*}=A x^{*} \in H_{2} \text { solves } 0 \in g\left(y^{*}\right)+B_{2}\left(y^{*}\right),
\end{aligned}
$$

where $A: H_{1} \rightarrow H_{2}$ is a bounded linear operator, $f: H_{1} \rightarrow H_{1}$ and $g: H_{2} \rightarrow H_{2}$ are given operators. In 2000, Moudafi [3] proposed the viscosity approximation method, which is formulated by considering the approximate well-posed problem and combining the non-expansive mapping $S$ with a contraction mapping $f$ on a non-empty, closed, and convex subset $\mathrm{C}$ of $H_{1}$. That is, given an arbitrary $x_{1}$ in $\mathrm{C}$, a sequence $\left\{x_{n}\right\}$ defined by

$$
x_{n+1}=\alpha_{n} f\left(x_{n}\right)+\left(1-\alpha_{n}\right) S x_{n}
$$

converges strongly to a point of $F(S)$, the set of fixed point of $S$, whenever $\left\{\alpha_{n}\right\} \subset(0,1)$ such that $\alpha_{n} \rightarrow 0$ as $n \rightarrow \infty$.

In $[4,5]$, the viscosity approximation method for split variational inclusion and the fixed point problem in a Hilbert space was presented as follows:

$$
\begin{aligned}
u_{n} & =J_{\lambda}^{B_{1}}\left(x_{n}+\gamma_{n} A^{*}\left(J_{\lambda}^{B_{2}}-I\right) A x_{n}\right) ; \\
x_{n+1} & =\alpha_{n} f\left(x_{n}\right)+\left(1-\alpha_{n}\right) T^{n}\left(u_{n}\right), \forall n \geq 1,
\end{aligned}
$$

where $B_{1}$ and $B_{2}$ are maximal monotone operators, $J_{\lambda}^{B_{1}}$ and $J_{\lambda}^{B_{2}}$ are resolvent mappings of $B_{1}$ and $B_{2}$, respectively, $f$ is the Meir Keeler function, $T$ a non-expansive mapping, and $A^{*}$ is the adjoint of $A$, $\gamma_{n}, \alpha_{n} \in(0,1)$ and $\lambda>0$.

The algorithm introduced by Schopfer et al. [6] involves computations in terms of Bregman distance in the setting of p-uniformly convex and uniformly smooth real Banach spaces. Their iterative algorithm given below converges weakly under some suitable conditions:

$$
x_{n+1}=\Pi_{C} J^{-1}\left(J x_{n}+\gamma A^{*} J\left(P_{Q}-I\right) A x_{n}\right), n \geq 0,
$$

where $\Pi_{C}$ denotes the Bregman projection and $P_{C}$ denotes metric projection onto $C$. However, strong convergence is more useful than the weak convergence in some applications. Recently, strong convergence theorems for the split feasibility problem (SFP) have been established in the setting of p-uniformly convex and uniformly smooth real Banach spaces [7-10].

Suppose that

$$
F(x, y)=f(x, y)+g(x, y)
$$

where $f, g: C \times C \longrightarrow \mathbb{R}$ are bifunctions on a closed and convex subset $C$ of a Banach space, which satisfy the following special properties $\left(A_{1}\right)-\left(A_{4}\right),\left(B_{1}\right)-\left(B_{3}\right)$ and $(C)$ : 


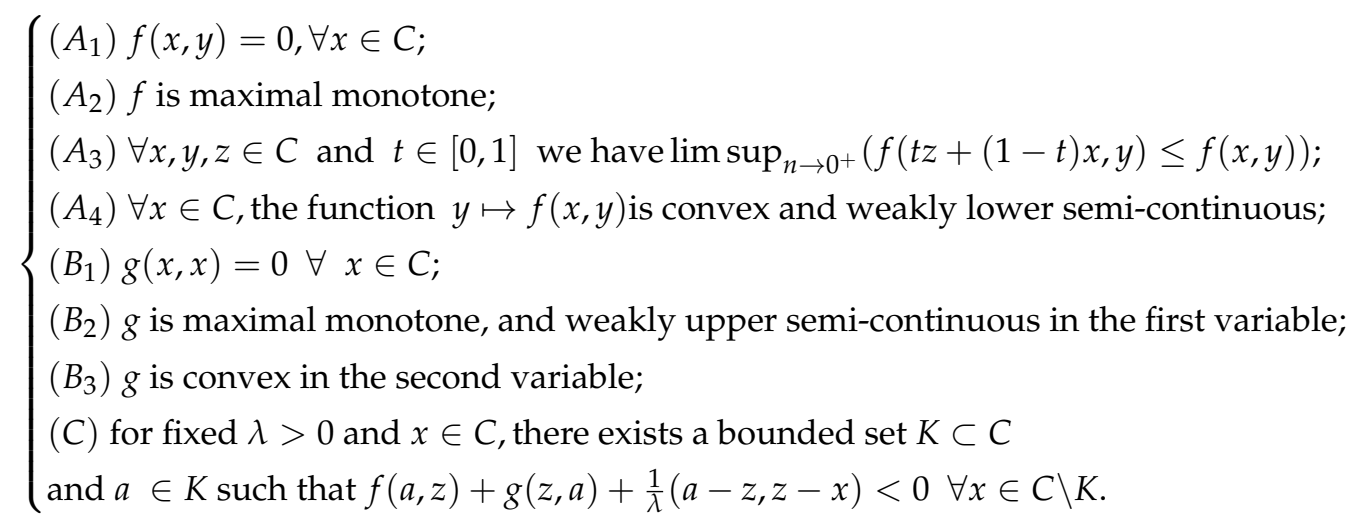

The well-known, generalized mixed equilibrium problem (GMEP) is to find an $x \in C$, such that

$$
F(x, y)+\langle B x, y-x\rangle \geq 0 \quad \forall \quad y \in C,
$$

where $B$ is nonlinear mapping.

In 2016, Payvand and Jahedi [11] introduced a new iterative algorithm for finding a common element of the set of solutions of a system of generalized mixed equilibrium problems, the set of common fixed points of a finite family of pseudo contraction mappings, and the set of solutions of the variational inequality for inverse strongly monotone mapping in a real Hilbert space. Their sequence is defined as follows:

$$
\left\{\begin{array}{l}
g_{i}\left(u_{n, i}, y\right)+\left\langle C_{i} u_{n, i}+S_{n, i} x_{n}, y-u_{n, i}\right\rangle+\theta_{i}(y)-\theta_{i}\left(u_{n, i}\right) \\
+\frac{1}{r_{n, i}}\left\langle y-u_{n, i}, u_{n, i}-x_{n}\right\rangle \geq 0 \forall y \in K, \forall i \in I, \\
y_{n}=\alpha_{n} v_{n}+\left(1-\alpha_{n}(I-f) P_{K}\left(\sum_{i=0}^{\infty} \delta_{n, i} u_{n, i}-\lambda_{n} A \sum_{i=0}^{\infty} \delta_{n, i} u_{n, i}\right),\right. \\
x_{n+1}=\beta_{n} x_{n}+\left(1+\beta_{n}\right)\left(\gamma_{0}+\sum_{j=1}^{\infty} \gamma_{j} T_{j}\right) P_{K}\left(y_{n}-\lambda_{n} A y_{n}\right) n \geq 1
\end{array}\right.
$$

where $g_{i}$ are bifunctions, $S_{i}$ are $\epsilon$ - inverse strongly monotone mappings, $C_{i}$ are monotone and Lipschtz continuous mappings, $\theta_{i}$ are convex and lower semicontinuous functions, $A$ is a $\Phi$ - inverse strongly monotone mapping, and $f$ is an $\iota$-contraction mapping and $\alpha_{n}, \delta_{n}, \beta_{n}, \lambda_{n}, \gamma_{0} \in(0,1)$.

In this paper, inspired by the above cited works, we use a modified version of (1), (2) and (4) to approximate a solution of the problem proposed here. Both the iterative methods and the underlying space used here are improvements and extensions of those employed in [2,6,7,9-11] and the references therein.

Let $p, q \in(1, \infty)$ be conjugate exponents, that is, $\frac{1}{p}+\frac{1}{q}=1$. For each $p>1$, let $g(t)=t^{p-1}$ be a gauge function where $g: \mathbb{R}^{+} \longrightarrow \mathbb{R}^{+}$with $g(0)=0$ and $\lim _{t \rightarrow \infty} g(t)=\infty$. We define the generalized duality map $J_{p}: E \longrightarrow 2^{E^{*}}$ by

$$
J_{g(t)}=J_{p}(x)=\left\{x^{*} \in E^{*} ;\left\langle x, x^{*}\right\rangle=\|x\|\left\|x^{*}\right\|,\left\|x^{*}\right\|=g(\|x\|)=\|x\|^{p-1}\right\} .
$$

In the sequel, $a \vee b$ denotes $\max \{a, b\}$.

Lemma 1 ([12]). In a smooth Banach space $E$, the Bregman distance $\triangle_{p}$ of $x$ to $y$, with respect to the convex continuous function $f: E \rightarrow R$, such that $f(x)=\frac{1}{p}\|x\|^{p}$, is defined by

$$
\triangle_{p}(x, y)=\frac{1}{q}\|x\|^{p}-\left\langle J^{p}(x), y\right\rangle+\frac{1}{p}\|y\|^{p},
$$

for all $x, y \in E$ and $p>1$. 
A Banach space $\mathrm{E}$ is said to be uniformly convex if, for $x, y \in E, 0<\delta_{E}(\epsilon) \leq 1$, where $\delta_{E}(\epsilon)=$ $\inf \left\{1-\left\|\frac{1}{2}(x+y)\right\| ;\|x\|=\|y\|=1,\|x-y\| \geq \epsilon\right.$, where $\left.0 \leq \epsilon \leq 2\right\}$.

Definition 1. A Banach space $E$ is said to be uniformly smooth, if for $x, y \in E, \lim _{r \rightarrow 0}\left(\frac{\rho_{E}(r)}{r}\right)=0$ where $\rho_{E}(r)=\frac{1}{2} \sup \left\{\|x+y\|+\|x-y\|-2:\|x\|=1,\|y\| \leq r ; 0 \leq r<\infty\right.$ and $\left.0 \leq \rho_{E}(r)<\infty\right\}$.

It is shown in [12] that:

1. $\rho_{E}$ is continuous, convex, and nondecreasing with $\rho_{E}(0)=0$ and $\rho_{E}(r) \leq r$

2. The function $r \mapsto \frac{\rho_{E}(r)}{r}$ is nondecreasing and fulfils $\frac{\rho_{E}(r)}{r}>0$ for all $r>0$.

Definition 2 ([13]). Let $E$ be a smooth Banach space. Let $\triangle_{p}$ be the Bregman distance. A mapping $T: E \longrightarrow E$ is said to be a strongly non-expansive left Bregman with respect to the non-empty fixed point set of $T, F(T)$, if $\triangle_{p}(T(x), v) \leq \triangle_{p}(x, v) \forall x \in E$ and $v \in F(T)$.

Furthermore, if $\left\{x_{n}\right\} \subset C$ is bounded and $\lim _{n \rightarrow \infty}\left(\triangle_{p}\left(x_{n}, v\right)-\triangle_{p}\left(T x_{n}, v\right)\right)=0$, then it follows that $\lim _{n \rightarrow \infty} \triangle_{p}\left(x_{n}, T x_{n}\right)=0$.

Definition 3. Let $E$ be a smooth Banach space. Let $\triangle_{p}$ be the Bregman distance. A mapping $T: E \longrightarrow E$ is said to be a strongly asymptotically non-expansive left Bregman with $\left\{k_{n}\right\} \subset[1, \infty)$ if there exists non-negative real sequences $\left\{k_{n}\right\}$ with $\lim _{n \rightarrow \infty} k_{n}=1$, such that $\triangle_{p}\left(T^{n}(x), T^{n}(v)\right) \leq k_{n} \triangle_{p}(x, v), \forall(x, v) \in E \times F(T)$.

Lemma 2 ([14]). Let E be a real uniformly convex Banach space, $K$ a non-empty closed subset of $E$, and $T$ : $K \rightarrow K$ an asymptotically non-expansive mapping. Then, $I-T$ is demi-closed at zero, if $\left\{x_{n}\right\} \subset K$ converges weakly to a point $p \in K$ and $\lim _{n \rightarrow \infty}\left\|T x_{n}-x_{n}\right\|=0$, then $p=T p$.

Lemma 3 ([12]). In a smooth Banach space E, let $x_{n} \in$ E. Consider the following assertions:

1. $\lim _{n \rightarrow \infty}\left\|x_{n}-x\right\|=0$

2. $\lim _{n \rightarrow \infty}\left\|x_{n}\right\|=\|x\|$ and $\lim _{n \rightarrow \infty}\left\langle J^{p}\left(x_{n}\right), x\right\rangle=\left\langle J^{p}(x), x\right\rangle$

3. $\lim _{n \rightarrow \infty} \triangle_{p}\left(x_{n}, x\right)=0$.

The implication (1) $\Longrightarrow(2) \Longrightarrow$ (3) are valid. If $E$ is also uniformly convex, then the assertions are equivalent.

Lemma 4. Let $E$ be a smooth Banach space. Let $\triangle_{p}$ and $V_{p}$ be the mappings defined by $\triangle_{p}(x, y)=\frac{1}{q}\|x\|^{p}-$ $\left\langle J_{E}^{p} x, y\right\rangle+\frac{1}{p}\|y\|^{p}$ for all $(x, y) \in E \times E$ and $V_{p}\left(x^{*}, x\right)=\frac{1}{q}\left\|x^{*}\right\|^{q}-\left\langle x^{*}, x\right\rangle+\frac{1}{p}\|x\|^{p}$ for all $\left(x, x^{*}\right) \in E \times E^{*}$. Then, $\triangle_{p}(x, y)=V_{p}\left(x^{*}, y\right)$ for all $x, y \in E$.

Lemma 5 ([12]). Let $E$ be a reflexive, strictly convex, and smooth Banach space, and $J^{p}$ be a duality mapping of $E$. Then, for every closed and convex subset $C \subset E$ and $x \in E$, there exists a unique element $\Pi_{C}^{p}(x) \in C$, such that $\triangle_{p}\left(x, \Pi_{C}^{p}(x)\right)=\min _{y \in C} \triangle_{p}(x, y)$; here, $\Pi_{C}^{p}(x)$ denotes the Bregman projection of $x$ onto $C$, with respect to the function $f(x)=\frac{1}{p}\|x\|^{p}$. Moreover, $x_{0} \in C$ is the Bregman projection of $x$ onto $C$ if

$$
\left\langle J^{p}\left(x_{0}-x\right), y-x_{0}\right\rangle \geq 0
$$

or equivalently

$$
\triangle_{p}\left(x_{0}, y\right) \leq \triangle_{p}(x, y)-\triangle_{p}\left(x, x_{0}\right) \text { for every } y \in C .
$$

Lemma 6 ( [15]). In the case of a uniformly convex space, $E$, with the duality map $J^{q}$ of $E^{*}, \forall x^{*}, y^{*} \in E^{*}$ we have

$$
\left\|x^{*}-y^{*}\right\|^{q} \leq\left\|x^{*}\right\|^{q}-q\left\langle J^{q}\left(x^{*}\right), y^{*}\right\rangle+\overline{\sigma_{q}}\left(x^{*}, y^{*}\right), \text { where }
$$




$$
\begin{aligned}
& \overline{\sigma_{q}}\left(x^{*}, y^{*}\right)=q G_{q} \int_{0}^{1} \frac{\left(\left\|x^{*}-t y^{*}\right\| \vee\left\|x^{*}\right\|\right)^{q}}{t} \rho_{E^{*}}\left(\frac{t\left\|y^{*}\right\|}{2\left(\left\|x^{*}-t y^{*}\right\| \vee\left\|x^{*}\right\|\right)}\right) d t \\
& \text { and } G_{q}=8 \vee 64 c K_{q}^{-1} \text { with } c, K_{q}>0 .
\end{aligned}
$$

Lemma 7 ([12]). Let $E$ be a reflexive, strictly convex, and smooth Banach space. If we write $\triangle_{q}^{*}(x, y)=$ $\frac{1}{p}\left\|x^{*}\right\|^{q}-\left\langle J_{E^{*}}^{q} x^{*}, y^{*}\right\rangle+\frac{1}{q}\left\|y^{*}\right\|^{q}$ for all $\left(x^{*}, y^{*}\right) \in E^{*} \times E^{*}$ for the Bregman distance on the dual space $E^{*}$ with respect to the function $f_{q}^{*}\left(x^{*}\right)=\frac{1}{q}\left\|x^{*}\right\|^{q}$, then we have $\triangle_{p}(x, y)=\triangle_{q}^{*}\left(x^{*}, y^{*}\right)$.

Lemma 8 ([16]). Let $\left\{\alpha_{n}\right\}$ be a sequence of non-negative real numbers, such that $\alpha_{n+1} \leq\left(1-\beta_{n}\right) \alpha_{n}+\delta_{n}$, $n \geq 0$, where $\left\{\beta_{n}\right\}$ is a sequence in $(0,1)$ and $\left\{\delta_{n}\right\}$ is a sequence in $R$, such that

1. $\lim _{n \rightarrow \infty} \beta_{n}=0, \sum_{n=1}^{\infty} \beta_{n}=\infty$;

2. $\underset{n \rightarrow \infty}{\limsup } \frac{\delta_{n}}{\beta_{n}} \leq 0$ or $\sum_{n=1}^{\infty}\left|\delta_{n}\right|<\infty$.

Then, $\lim _{n \rightarrow \infty} \alpha_{n}=0$.

Lemma 9. Let $E$ be reflexive, smooth, and strictly convex Banach space. Then, for all $x, y, z \in E$ and $x^{*}, z^{*} \in E^{*}$ the following facts hold:

1. $\triangle_{p}(x, y) \geq 0$ and $\triangle_{p}(x, y)=0$ iff $x=y$;

2. $\triangle_{p}(x, y)=\triangle_{p}(x, z)+\triangle_{p}(z, y)+\left\langle x^{*}-z^{*}, z-y\right\rangle$.

Lemma 10 ([17]). Let E be a real uniformly convex Banach space. For arbitrary $r>1$, let $B_{r}(0)=\{x \in E$ : $\|x\| \leq r\}$. Then, there exists a continuous strictly increasing convex function

$$
g:[0, \infty) \longrightarrow[0, \infty), g(0)=0
$$

such that for every $x, y \in B_{r}(0), f_{x} \in J_{p}(x), f_{y} \in J_{p}(y)$ and $\lambda \in[0,1]$, the following inequalities hold:

$$
\|\lambda x+(1-\lambda) y\|^{p} \leq \lambda\|x\|^{p}+(1-\lambda)\|y\|^{p}-\left(\lambda^{p}(1-\lambda)+(1-\lambda)^{p} \lambda\right) g(\|x-y\|)
$$

and

$$
\left\langle x-y, f_{x}-f_{y}\right\rangle \geq g(\|x-y\|) .
$$

Lemma 11 ([18]). Suppose that $\sum_{n=1}^{\infty} \sup \left\{\left\|T_{n+1} z-T_{n} z\right\|: z \in C\right\}<\infty$. Then, for each $y \in C,\left\{T_{n} y\right\}$ converges strongly to some point of $C$. Moreover, let $T$ be a mapping of $C$ onto itself, defined by $T y=\lim _{n \rightarrow \infty} T_{n} y$ for all $y \in C$. Then, $\lim _{n \rightarrow \infty} \sup \left\{\left\|T z-T_{n} z\right\|: z \in C\right\}=0$. Consequently, by Lemma 3, $\lim _{n \rightarrow \infty} \sup \left\{\triangle_{p}\left(T z, T_{n} z\right)\right.$ : $z \in C\}=0$.

Lemma 12 ([19]). Let E be a reflexive, strictly convex, and smooth Banach space, and $C$ be a non-empty, closed convex subset of $E$. If $f, g: C \times C \longrightarrow \mathbb{R}$ be two bifunctions which satisfy the conditions $\left(A_{1}\right)-$ $\left(A_{4}\right),\left(B_{1}\right)-\left(B_{3}\right)$ and $(C)$, in (3), then for every $x \in E$ and $r>0$, there exists a unique point $z \in C$ such that $f(z, y)+g(z, y)+\frac{1}{r}\langle y-z, j z-j x\rangle \geq 0 \forall y \in C$.

For $f(x)=\frac{1}{p}\|x\|^{p}$, Reich and Sabach [20] obtained the following technical result:

Lemma 13. Let $E$ be a reflexive, strictly convex, and smooth Banach space, and $C$ be a non-empty, closed, and convex subset of $E$. Let $f, g: C \times C \longrightarrow \mathbb{R}$ be two bifunctions which satisfy the conditions $\left(A_{1}\right)-$ 
$\left(A_{4}\right),\left(B_{1}\right)-\left(B_{3}\right)$ and $(C)$, in (3). Then, for every $x \in E$ and $r>0$, we define a mapping $S_{r}: E \longrightarrow C$ as follows;

$$
S_{r}(x)=\left\{z \in C: f(z, y)+g(z, y)+\frac{1}{r}\left\langle y-z, J_{E}^{p} z-J_{E}^{p} x\right\rangle \geq 0 \forall y \in C\right\} .
$$

Then, the following conditions hold:

1. $S_{r}$ is single-valued;

2. $S_{r}$ is a Bregman firmly non-expansive-type mapping, that is,

$$
\forall x, y \in E\left\langle S_{r} x-S_{r} y, J_{E}^{p} S_{r} x-J_{E}^{p} S_{r} y\right\rangle \leq\left\langle S_{r} x-S_{r} y, J_{E}^{p} x-J_{E}^{p} y\right\rangle
$$

or equivalently

$$
\triangle_{p}\left(S_{r} x, S_{r} y\right)+\triangle_{p}\left(S_{r} y, S_{r} x\right)+\triangle_{p}\left(S_{r} x, x\right)+\triangle_{p}\left(S_{r} y, y\right) \leq \triangle_{p}\left(S_{r} x, y\right)+\triangle_{p}\left(S_{r} y, x\right) ;
$$

3. $\quad F\left(S_{r}\right)=M E P(f, g)$, here $M E P$ stands for mixed equilibrium problem;

4. $M E P(f, g)$ is closed and convex;

5. for all $x \in E$ and for all $v \in F\left(S_{r}\right), \triangle_{p}\left(v, S_{r} x\right)+\triangle_{p}\left(S_{r} x, x\right) \leq \triangle_{p}(v, x)$.

\section{Main Results}

Let $E_{1}$ and $E_{2}$ be uniformly convex and uniformly smooth Banach spaces and $E_{1}^{*}$ and $E_{2}^{*}$ be their duals, respectively. For $i \in I$, let $U_{i}: E_{1} \rightarrow 2^{E_{1}^{*}}$ and $T_{i}: E_{2} \rightarrow 2^{E_{2}^{*}}, i \in I$ be multi-valued maximal monotone operators. For $i \in I, \delta>0, p, q \in(1, \infty)$ and $K \subset E_{1}$ closed and convex, let $\Phi_{i}: K \times K \rightarrow \mathbb{R}, i \in I$, be bifunctions satisfying $(A 1)-(A 4)$ in (3), let $B_{\delta}^{U_{i}}: E_{1} \rightarrow E_{1}$ be resolvent operators defined by $B_{\delta}^{U_{i}}=\left(J_{E_{1}}^{p}+\delta U_{i}\right)^{-1} J_{E_{1}}^{p}$ and $B_{\delta}^{T_{i}}: E_{2} \rightarrow E_{2}$ be resolvent operators defined by $B_{\delta}^{T_{i}}=\left(J_{E_{2}}^{p}+\delta T_{i}\right)^{-1} J_{E_{2}}^{p}$. Let $A: E_{1} \rightarrow E_{2}$ be a bounded and linear operator, $A^{*}$ denotes the adjoint of $A$ and $A K$ be closed and convex. For each $i \in I$, let $S_{i}: E_{1} \rightarrow E_{1}$ be a uniformly continuous Bregman asymptotically non-expansive operator with the sequences $\left\{k_{n, i}\right\} \subset[1, \infty)$ satisfying $\lim _{n \rightarrow \infty} k_{n, i}=1$. Denote by $Y: E_{1}^{*} \rightarrow E_{1}^{*}$ a firmly non-expansive mapping. Suppose that, for $i \in I, \theta_{i}: K \rightarrow R$ are convex and lower semicontinuous functions, $G_{i}: K \rightarrow E_{1}$ are $\varepsilon$ - inverse strongly monotone mappings and $C_{i}: K \rightarrow E_{1}$, are monotone and Lipschitz continuous mappings. Let $f: E_{1} \rightarrow E_{1}$ be a $\zeta$-contraction mapping, where $\zeta \in(0,1)$. Suppose that $\Pi_{A K}^{p}: E_{2} \rightarrow A K$ is a generalized Bregman projection onto $A K$. Let $\Omega=\left\{x^{*} \in \cap_{i=1}^{\infty} \operatorname{SOLVIP}\left(U_{i}\right) ; A x^{*} \in \cap_{i=1}^{\infty} \operatorname{SOLVIP}\left(T_{i}\right)\right\}$ be the set of solution of the split variational inclusion problem, $\omega=\left\{x^{*} \in \cap_{i=1}^{\infty} \operatorname{GMEP}\left(G_{i}, C_{i}, \theta_{i}, g_{i}\right)\right\}$ be the solution set of a system of generalized mixed equilibrium problems, and $\Im=\left\{x^{*} \in \cap_{i=1}^{\infty} F\left(S_{i}\right)\right\}$ be the common fixed-point set of $S_{i}$ for each $i \in I$. Let the sequence $\left\{x_{n}\right\}$ be defined as follows:

$$
\left\{\begin{array}{l}
\Phi_{i}\left(u_{n, i}, y\right)+\left\langle J_{E_{1}}^{p} G_{n, i} x_{n}, y-u_{n, i}\right\rangle+\frac{1}{r_{n, i}}\left\langle y-u_{n, i}, J_{E_{1}}^{p} u_{n, i}-J_{E_{1}}^{p} x_{n}\right\rangle \geq 0 \forall y \in K, \\
\forall i \in I, \\
x_{n+1}=J_{E_{1}^{*}}^{q}\left(\sum_{i=0}^{\infty} \alpha_{n, i} B_{\delta_{n}}^{U_{i}}\left(J_{E_{1}}^{p} x_{n}-\sum_{i=0}^{\infty} \beta_{n, i} \lambda_{n} A^{*} J_{E_{2}}^{p}\left(I-\Pi_{A K}^{p} B_{\delta_{n}}^{T_{i}}\right) A u_{n, i}\right)\right),
\end{array}\right.
$$

where $\Phi_{i}(x, y)=g_{i}(x, y)+\left\langle J_{E_{1}}^{p} C_{i} x, y-x\right\rangle+\theta_{i}(y)-\theta_{i}(x)$.

We shall strictly employ the above terminology in the sequel.

Lemma 14. Suppose that $\bar{\sigma}_{q}$ is the function (5) in Lemma 6 for the characteristic inequality of the uniformly smooth dual $E_{1}^{*}$. For the sequence $\left\{x_{n}\right\} \subset E_{1}$ defined by (7), let $0 \neq x_{n} \in E_{1}, 0 \neq A, 0 \neq J_{E_{1}}^{p} G_{n, i} x_{n} \in E_{1}^{*}$ and $0 \neq \sum_{i=0}^{\infty} \beta_{n, i} J_{E_{2}}^{p}\left(I-\Pi_{A K}^{p} B_{\delta_{n}}^{T_{i}}\right) A u_{n, i} \in E_{2}^{*}, i \in I$. Let , for $\lambda_{n, i}>0$ and $r_{n, i}>0, i \in I$ be defined by

$$
\begin{aligned}
\lambda_{n, i} & =\frac{1}{\|A\|} \frac{1}{\left\|\sum_{i=0}^{\infty} \beta_{n, i} I_{E_{2}}^{p}\left(I-\Pi_{A K}^{p} B_{\delta_{n}}^{T_{i}}\right) A u_{n, i}\right\|}, \text { and } \\
r_{n, i} & =\frac{1}{\left\|J_{E_{1}}^{p} G_{n, i} x_{n}\right\|}, \text { respectively. }
\end{aligned}
$$


Then for $\mu_{n, i}=\frac{1}{\left\|x_{n}\right\|^{p-1}}$,

$$
2^{q} G_{q}\left\|J_{E_{1}}^{p} x_{n}\right\|^{p} \rho_{E_{1}^{*}}\left(\mu_{n, i}\right) \geq\left\{\begin{array}{l}
\frac{1}{q} \overline{\sigma_{q}}\left(J_{E_{1}}^{p} x_{n}, r_{n, i} J_{E_{1}}^{p} G_{n, i} x_{n}\right) \\
\frac{1}{q} \overline{\sigma_{q}}\left(J_{E_{1}}^{p} x_{n}, \sum_{i=0}^{\infty} \beta_{n, i} \lambda_{n} A^{*} \sum_{i=0}^{\infty} \beta_{n, i} J_{E_{2}}^{p}\left(I-\Pi_{A K}^{p} B_{\delta_{n}}^{T_{i}}\right) A u_{n, i}\right),
\end{array}\right.
$$

where $G_{q}$ is the constant defined in Lemma 6 and $\rho_{E_{1}^{*}}$ is the modulus of smoothness of $E_{1}^{*}$.

Proof. By Lemma 12, (6) in Lemma 13 and (7), for each $i \in I$, we have that $u_{n, i}=J_{E_{1}^{*}}^{q}\left(\mathrm{Y}_{r_{n, i}}\left(J_{E_{1}}^{p} x_{n}-\right.\right.$ $\left.\left.r_{n, i} J_{E_{1}}^{p} G_{n, i} x_{n}\right)\right)$. By Lemma 6, we get

$$
\begin{gathered}
\frac{1}{q} \bar{\sigma}_{q}\left(J_{E_{1}}^{p} x_{n}, r_{n, i} J_{E_{1}}^{p} G_{n, i} x_{n}\right)=G_{q} \int_{0}^{1} \frac{\left(\left\|J_{E_{1}}^{p} x_{n}-t r_{n, i} J_{E_{1}}^{p} G_{n, i} x_{n}\right\| \vee\left\|J_{E_{1}}^{p} x_{n}\right\|\right)^{q}}{t} \times \\
\rho_{E^{*}}\left(\frac{t\left\|r_{n, i} J_{E_{1}}^{p} G_{n, i} x_{n}\right\|}{\left(\left\|J_{E_{1}}^{p} x_{n}-t r_{n, i} J_{E_{1}}^{p} G_{n, i} x_{n}\right\| \vee\left\|J_{E_{1}}^{p} x_{n}\right\|\right)}\right) d t, \\
\text { for every } t \in[0,1] .
\end{gathered}
$$

However, by (9) and Definition 1(2), we have

$$
\begin{aligned}
\rho_{E_{1}^{*}}\left(\frac{t\left\|r_{n, i} J_{E_{1}}^{p} G_{n, i} x_{n}\right\|}{\left(\left\|J_{E_{1}}^{p} x_{n}-t r_{n, i} J_{E_{1}}^{p} G_{n, i} x_{n}\right\| \vee\left\|J_{E_{1}}^{p} x_{n}\right\|\right)}\right) & \leq \rho_{E_{1}^{*}}\left(\frac{t\left\|r_{n, i} J_{E_{1}}^{p} G_{n, i} x_{n}\right\|}{\left\|x_{n}\right\|^{p-1}}\right) \\
& =\rho_{E_{1}^{*}}\left(t \mu_{n, i}\right) .
\end{aligned}
$$

Substituting (12) into (11), and using the nondecreasing of function $\rho_{E_{1}^{*}}$, we have

$$
\frac{1}{q} \overline{\sigma_{q}}\left(J_{E_{1}}^{p} x_{n}, r_{n, i} J_{E_{1}}^{p} G_{n, i} x_{n}\right) \leq 2^{q} G_{q}\left\|x_{n}\right\|^{p} \rho_{E_{1}^{*}}\left(\mu_{n, i}\right)
$$

In addition, by Lemma 6, we have

$$
\begin{gathered}
\frac{1}{q} \overline{\sigma_{q}}\left(J_{E_{1}}^{p} x_{n}, \sum_{i=0}^{\infty} \beta_{n, i} \lambda_{n} A^{*} J_{E_{2}}^{p}\left(I-\Pi_{A K}^{p} B_{\delta_{n}}^{T_{i}}\right) A u_{n, i}\right) \\
=G_{q} \int_{0}^{1} \frac{\left(\left\|J_{E_{1}}^{p} x_{n}-\sum_{i=0}^{\infty} \beta_{n, i} \lambda_{n} A^{*} J_{E_{2}}^{p}\left(I-\Pi_{A K}^{p} B_{\delta_{n}}^{T_{i}}\right) A u_{n, i}\right\| \vee\left\|J_{E_{1}}^{p} x_{n}\right\|\right)^{q}}{t} \times \\
\rho_{E^{*}}\left(\frac{t\left\|\sum_{i=0}^{\infty} \beta_{n, i} \lambda_{n} A^{*} J_{E_{2}}^{p}\left(I-\Pi_{A K}^{p} B_{\delta_{n}}^{T_{i}}\right) A u_{n, i}\right\|}{\left(\left\|J_{E_{1}}^{p} x_{n}-\sum_{i=0}^{\infty} \beta_{n, i} \lambda_{n} A^{*} J_{E_{2}}^{p}\left(I-\Pi_{A K}^{p} B_{\delta_{n}}^{T_{i}}\right) A u_{n, i}\right\| \vee\left\|J_{E_{1}}^{p} x_{n}\right\|\right)}\right) d t \\
\text { for every } t \in[0,1] .
\end{gathered}
$$

However, by (8) and Definition 1(2), we have

$$
\begin{aligned}
& \rho_{E_{1}^{*}}\left(\frac{t\left\|\sum_{i=0}^{\infty} \beta_{n, i} \lambda_{n} A^{*} J_{E_{2}}^{p}\left(I-\Pi_{A K}^{p} B_{\delta_{n}}^{T_{i}}\right) A u_{n, i}\right\|}{\left(\left\|J_{E_{1}}^{p} x_{n}-t \sum_{i=0}^{\infty} \beta_{n, i} \lambda_{n} A^{*} J_{E_{2}}^{p}\left(I-\Pi_{A K}^{p} B_{\delta_{n}}^{T_{i}}\right) A u_{n, i}\right\| \vee\left\|J_{E_{1}}^{p} x_{n}\right\|\right)}\right) \\
& \quad \leq \rho_{E_{1}^{*}}\left(\frac{t\left\|\sum_{i=0}^{\infty} \beta_{n, i} \lambda_{n, i} A^{*} J_{E_{2}}^{p}\left(I-\Pi_{A K}^{p} B_{\delta_{n}}^{T_{i}}\right) A u_{n, i}\right\|}{\left\|x_{n}\right\|^{p-1}}\right)=\rho_{E_{1}^{*}}\left(t \mu_{n, i}\right) .
\end{aligned}
$$




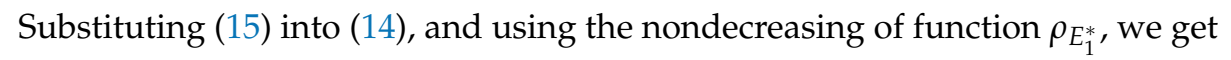

$$
\begin{aligned}
& \frac{1}{q} \overline{\sigma_{q}}\left(J_{E_{1}}^{p} x_{n}, \sum_{i=0}^{\infty} \beta_{n, i} \lambda_{n} A^{*} J_{E_{2}}^{p}\left(I-\Pi_{A K}^{p} B_{\delta_{n}}^{T_{i}}\right) A u_{n, i}\right) \\
& \quad \leq 2^{q} G_{q}\left\|x_{n}\right\|^{p} \rho_{E_{1}^{*}}\left(\mu_{n, i}\right) .
\end{aligned}
$$

By (13) and (16), the result follows.

Lemma 15. For the sequence $\left\{x_{n}\right\} \subset E_{1}$, defined by $(7), i \in I$, let $0 \neq \sum_{i=0}^{\infty} \beta_{n, i} J_{E_{2}}^{p}\left(I-\Pi_{A K}^{p} B_{\delta_{n}}^{T_{i}}\right) A u_{n, i} \in E_{2}^{*}$, $0 \neq J_{E_{1}}^{p} G_{n, i} x_{n} \in E_{1}^{*}$, and $\lambda_{n}>0$ and $r_{n, i}>0, i \in I$, be defined by

$$
\lambda_{n}=\frac{1}{\|A\|} \frac{1}{\left\|\sum_{i=0}^{\infty} \beta_{n, i} i_{E_{2}}^{p}\left(I-\Pi_{A K}^{p} B_{\delta_{n}}^{T_{i}}\right) A u_{n, i}\right\|}
$$

and

$$
r_{n, i}=\frac{1}{\left\|J_{E_{1}}^{p} G_{n, i} x_{n}\right\|}
$$

where $\iota, \gamma \in(0,1)$ and $\mu_{n, i}=\frac{1}{\left\|x_{n}\right\|^{p-1}}$ are chosen such that

$$
\rho_{E_{1}^{*}}\left(\mu_{n, i}\right)=\frac{\iota}{{ }^{q} G_{q}\|A\|} \times \frac{\left\|\sum_{i=0}^{\infty} \beta_{n, i} J_{E_{2}}^{p}\left(I-\Pi_{A K}^{p} B_{\delta_{n}}^{T_{i}}\right) A u_{n, i}\right\|^{p}}{\left\|x_{n}\right\|^{p}\left\|\sum_{i=0}^{\infty} \beta_{n, i} J_{E_{2}}^{p}\left(I-\Pi_{A K}^{p} B_{\delta_{n}}^{T_{i}}\right) A u_{n, i}\right\|^{p-1}},
$$

and

$$
\rho_{E_{1}^{*}}\left(\mu_{n, i}\right)=\frac{\gamma\left\langle J_{E_{1}}^{p} G_{n, i} x_{n}, x_{n}-v\right\rangle}{2^{q} G_{q}\left\|x_{n}\right\|^{p}\left\|J_{E_{1}}^{p} G_{n, i} x_{n}\right\|} .
$$

Then, for all $v \in \Gamma$, we get

$$
\begin{aligned}
& \triangle_{p}\left(x_{n+1}, v\right) \leq \triangle_{p}\left(x_{n}, v\right) \\
&-[1-\iota] \times \frac{\left\langle\sum_{i=0}^{\infty} \beta_{n, i} J_{E_{2}}^{p}\left(I-\Pi_{A K}^{p} B_{\delta_{n}}^{T_{i}}\right) A u_{n, i} \sum_{i=0}^{\infty} \beta_{n, i}\left(I-\Pi_{A K}^{p} B_{\delta_{n}}^{T_{i}}\right) A u_{n, i}\right\rangle}{\|A\|\left\|\sum_{i=0}^{\infty} \beta_{n, i} J_{E_{2}}^{p}\left(I-\Pi_{A K}^{p} B_{\delta_{n}}^{T_{i}}\right) A u_{n, i}\right\|}
\end{aligned}
$$

and

$$
\triangle_{p}\left(u_{n}, v\right) \leq \triangle_{p}\left(x_{n}, v\right)-[1-\gamma] \times \frac{\left\langle J_{E_{1}}^{p} G_{n, i} x_{n}, x_{n}-v\right\rangle}{\left\|J_{E_{1}}^{p} G_{n, i} x_{n}\right\|}, \text { respectively }
$$

Proof. By Lemmas 13,4 and 6, for each $i \in I$, we get that $u_{n, i}=J_{E_{1}^{*}}^{q}\left(Y_{r_{n, i}}\left(J_{E_{1}}^{p} x_{n}-r_{n, i} J_{E_{1}}^{p} G_{n, i} x_{n}\right)\right)$, and hence it follows that

$$
\begin{aligned}
\triangle_{p}\left(u_{n, i}, v\right) & \leq V_{p}\left(J_{E_{1}}^{p} x_{n}-r_{n, i} J_{E_{1}}^{p} G_{n, i} x_{n}, v\right) \\
& =-\left\langle J_{E_{1}}^{p} x_{n}, v\right\rangle+r_{n, i}\left(J_{E_{1}}^{p} G_{n, i} x_{n}, v\right\rangle \\
& +\frac{1}{q}\left\|J_{E_{1}}^{p} x_{n}-r_{n, i} J_{E_{1}}^{p} G_{n, i} x_{n}\right\|^{q}+\frac{1}{p}\|v\|^{p} .
\end{aligned}
$$


By Lemmas 6 and 14, we have

$$
\begin{aligned}
& \frac{1}{q}\left\|J_{E_{1}}^{p} x_{n}-r_{n, i} J_{E_{1}}^{p} G_{n, i} x_{n}\right\|^{q} \\
& \quad \leq \frac{1}{q}\left\|J_{E_{1}}^{p} x_{n}\right\|^{q}-r_{n, i}\left\langle J_{E_{1}}^{p} G_{n, i} x_{n}, x_{n}\right\rangle+2^{q} G_{q}\left\|J_{E_{1}}^{p} x_{n}\right\|^{p} \rho_{E_{1}^{*}}\left(\mu_{n, i}\right) .
\end{aligned}
$$

Substituting (24) into (23), we have, by Lemma 4

$$
\begin{aligned}
& \triangle_{p}\left(u_{n, i}, v\right) \leq \triangle_{p}\left(x_{n}, v\right)+2^{q} G_{q}\left\|J_{E_{1}}^{p} x_{n}\right\|^{p} \rho_{E_{1}^{*}}\left(\mu_{n, i}\right) \\
& \quad-r_{n, i}\left\langle J_{E_{1}}^{p} G_{n, i} x_{n}, x_{n}-v\right\rangle
\end{aligned}
$$

Substituting (18) and (20) into (25), we have

$$
\begin{aligned}
& \triangle_{p}\left(u_{n, i}, v\right) \leq \triangle_{p}\left(x_{n}, v\right)+\frac{\gamma\left\langle J_{E_{1}}^{p} G_{n, i} x_{n}, x_{n}-v\right\rangle}{\left\|J_{E_{1}}^{p} G_{n, i} x_{n}\right\|}-\frac{\left\langle J_{E_{1}}^{p} G_{n, i} x_{n}, x_{n}-v\right\rangle}{\left\|J_{E_{1}}^{p} G_{n, i} x_{n}\right\|} \\
& =\triangle_{p}\left(x_{n}, v\right)-[1-\gamma] \times \frac{\left\langle J_{E_{1}}^{p} G_{n, i} x_{n}, x_{n}-v\right\rangle}{\left\|J_{E_{1}}^{p} G_{n, i} x_{n}\right\|} .
\end{aligned}
$$

Thus, (22) holds.

Now, for each $i \in I$, let $v=B_{\gamma}^{U_{i}} v$ and $A v=B_{\gamma}^{T_{i}} A v$. By Lemma 4, we have

$$
\begin{aligned}
& \triangle_{p}\left(y_{n}, v\right) \leq \frac{1}{q}\left\|J_{E_{1}}^{p} u_{n, i}-\sum_{i=0}^{\infty} \beta_{n, i} \lambda_{n} A^{*} J_{E_{2}}^{p}\left(I-\Pi_{A K}^{p} B_{\delta_{n}}^{T_{i}}\right) A u_{n, i}\right\|^{q}+\frac{1}{p}\|v\|^{p} \\
& -\left\langle J_{E_{1}}^{p} u_{n, i}, v\right\rangle+\left\langle\sum_{i=0}^{\infty} \beta_{n, i} \lambda_{n} A^{*} J_{E_{2}}^{p}\left(I-\Pi_{A K}^{p} B_{\delta_{n}}^{T_{i}}\right) A u_{n, i}, v\right\rangle,
\end{aligned}
$$

where,

$$
\begin{aligned}
& \left\langle\sum_{i=0}^{\infty} \beta_{n, i} \lambda_{n} A^{*} J_{E_{2}}^{p}\left(I-\Pi_{A K}^{p} B_{\delta_{n}}^{T_{i}}\right) A u_{n, i}, v\right\rangle \\
& =-\left\langle\sum_{i=0}^{\infty} \beta_{n, i} \lambda_{n} J_{E_{2}}^{p}\left(\Pi_{A K}^{p} B_{\delta_{n}}^{T_{i}}-I\right) A u_{n, i}\left(A v-\sum_{i=0}^{\infty} \beta_{n, i} A u_{n, i}\right)-\sum_{i=0}^{\infty} \beta_{n, i}\left(\Pi_{A K}^{p} B_{\delta_{n}}^{T_{i}}-I\right) A u_{n, i}\right\rangle \\
& -\left\langle\sum_{i=0}^{\infty} \beta_{n, i} \lambda_{n} J_{E_{2}}^{p}\left(I-\Pi_{A K}^{p} B_{\delta_{n}}^{T_{i}}\right) A u_{n, i}, \sum_{i=0}^{\infty} \beta_{n, i}\left(I-\Pi_{A K}^{p} B_{\delta_{n}}^{T_{i}}\right) A u_{n, i}\right\rangle \\
& +\left\langle\sum_{i=0}^{\infty} \beta_{n, i} \lambda_{n} J_{E_{2}}^{p}\left(I-\Pi_{A K}^{p} B_{\delta_{n}}^{T_{i}}\right) A u_{n, i}, A u_{n, i}\right\rangle .
\end{aligned}
$$

As $A K$ is closed and convex, by Lemma 5 and the variational inequality for the Bregman projection of zero onto $A K-\sum_{i=0}^{\infty} \beta_{n, i} A u_{n, i}$, we arrive at

$$
\left\langle\sum_{i=0}^{\infty} \beta_{n, i} \lambda_{n} J_{E_{2}}^{p}\left(\Pi_{A K}^{p} B_{\delta_{n}}^{T_{i}}-I\right) A u_{n, i}\left(A v-\sum_{i=0}^{\infty} \beta_{n, i} A u_{n, i}\right)-\sum_{i=0}^{\infty} \beta_{n, i}\left(\Pi_{A K}^{p} B_{\delta_{n}}^{T_{i}}-I\right) A u_{n, i}\right\rangle \geq 0
$$


and therefore,

$$
\begin{aligned}
& \left\langle\sum_{i=0}^{\infty} \beta_{n, i} \lambda_{n} A^{*} J_{E_{2}}^{p}\left(I-\Pi_{A K}^{p} B_{\delta_{n}}^{T_{i}}\right) A u_{n, i}, v\right\rangle \\
& \leq-\left\langle\sum_{i=0}^{\infty} \beta_{n, i} \lambda_{n} J_{E_{2}}^{p}\left(I-\Pi_{A K}^{p} B_{\delta_{n}}^{T_{i}}\right) A u_{n, i}, \sum_{i=0}^{\infty} \beta_{n, i}\left(I-\Pi_{A K}^{p} B_{\delta_{n}}^{T_{i}}\right) A u_{n, i}\right\rangle \\
& +\left\langle\sum_{i=0}^{\infty} \beta_{n, i} \lambda_{n} J_{E_{2}}^{p}\left(I-\Pi_{\Gamma}^{p} B_{\delta_{n}}^{T_{i}}\right) A u_{n, i}, A u_{n, i}\right\rangle .
\end{aligned}
$$

By Lemma 6, 14 and (27), we get

$$
\begin{aligned}
& \triangle_{p}\left(y_{n}, v\right) \leq \triangle_{p}\left(u_{n, i}, v\right)+2^{p} G_{p}\left\|J_{E_{1}}^{p} u_{n, i}\right\|^{p} \rho_{E_{1}^{*}}\left(\tau_{n, i}\right) \\
& \quad-\left\langle\sum_{i=0}^{\infty} \beta_{n, i} \lambda_{n} J_{E_{2}}^{p}\left(I-\Pi_{A K}^{p} B_{\delta_{n}}^{T_{i}}\right) A u_{n, i} \sum_{i=0}^{\infty} \beta_{n, i}\left(I-\Pi_{A K}^{p} B_{\delta_{n}}^{T_{i}}\right) A u_{n, i}\right\rangle .
\end{aligned}
$$

Substituting (17) and (19) into (28), we have

$$
\begin{aligned}
& \triangle_{p}\left(y_{n}, v\right) \leq \triangle_{p}\left(u_{n, i}, v\right)-[1-\imath] \\
& \quad \times \frac{\left\langle\sum_{i=0}^{\infty} \beta_{n, i} J_{E_{2}}^{p}\left(I-\Pi_{A K}^{p} B_{\delta_{n}}^{T_{i}}\right) A u_{n, i}, \sum_{i=0}^{\infty} \beta_{n, i}\left(I-\Pi_{A K}^{p} B_{\delta_{n}}^{T_{i}}\right) A u_{n, i}\right\rangle}{\|A\|\left\|\sum_{i=0}^{\infty} \beta_{n, i} J_{E_{2}}^{p}\left(I-\Pi_{A K}^{p} B_{\delta_{n}}^{T_{i}}\right) A u_{n, i}\right\|} .
\end{aligned}
$$

Thus, (21) holds as desired.

We now prove our main result.

Theorem 1. Let $g_{i}: K \times K \rightarrow R, i \in I$, be bifunctions satisfying $(A 1)-(A 4)$ in (3). For $\delta>0$ and $p, q \in(1, \infty)$, let $\left(I-\Pi_{A K}^{p} B_{\delta}^{T_{i}}\right), i \in I$, be demi-closed at zero. Let $x_{1} \in E_{1}$ be chosen arbitrarily and the sequence $\left\{x_{n}\right\}$ be defined as follows;

$$
\left\{\begin{array}{l}
g_{i}\left(u_{n, i}, y\right)+\left\langle J_{E_{1}}^{p} C_{i} u_{n, i}+J_{E_{1}}^{p} G_{n, i} x_{n}, y-u_{n, i}\right\rangle+\theta_{i}(y)-\theta_{i}\left(u_{n, i}\right) \\
+\frac{1}{r_{n, i}}\left\langle y-u_{n, i}, J_{E_{1}}^{p} u_{n, i}-J_{E_{1}}^{p} x_{n}\right\rangle \geq 0 \forall y \in K, \forall i \in I \\
y_{n}=J_{E_{1}^{*}}^{q}\left(\sum_{i=0}^{\infty} \alpha_{n, i} B_{\delta_{n}}^{U_{i}}\left(J_{E_{1}}^{p} u_{n, i}-\sum_{i=0}^{\infty} \beta_{n, i} \lambda_{n} A^{*} J_{E_{2}}^{p}\left(I-\Pi_{A K}^{p} B_{\delta_{n}}^{T_{i}}\right) A u_{n, i}\right)\right), \\
x_{n+1}=J_{E_{1}^{*}}^{q}\left(\eta_{n, 0} J_{E_{1}}^{p}\left(f\left(x_{n}\right)\right)+\sum_{i=1}^{\infty} \eta_{n, i} J_{E_{1}}^{p}\left(S_{n, i}\left(y_{n}\right)\right)\right) n \geq 1,
\end{array}\right.
$$

where $r_{n, i}=\frac{1}{\left\|J_{E_{1}}^{p} G_{n, i} x_{n}\right\|^{\prime}}, \mu_{n, i}=\frac{1}{\left\|x_{n}\right\|^{p-1}}$ and $\gamma \in(0,1)$ such that $\rho_{E_{1}^{*}}\left(\mu_{n, i}\right)=\frac{\gamma\left\langle J_{E_{1}}^{p} G_{n, i} x_{n}, x_{n}-v\right\rangle}{{ }^{2 q} G_{q}\left\|x_{n}\right\|^{p}\left\|J_{E_{1}}^{p} G_{n, i} x_{n}\right\|^{\prime}}$

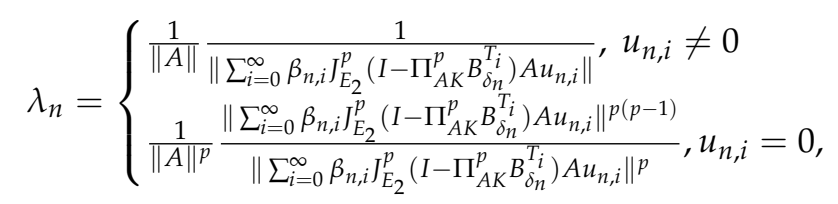

$\iota \in(0,1)$ and $\tau_{n, i}=\frac{1}{\left\|u_{n, i}\right\|^{p-1}}$ are chosen such that

$$
\rho_{E_{1}^{*}}\left(\tau_{n, i}\right)=\frac{\iota}{2^{q} G_{q}\|A\|} \times \frac{\left\|\sum_{i=0}^{\infty} \beta_{n, i} J_{E_{2}}^{p}\left(I-\Pi_{A K}^{p} B_{\delta_{n}}^{T_{i}}\right) A u_{n, i}\right\|^{p}}{\left\|u_{n, i}\right\|^{p}\left\|\sum_{i=0}^{\infty} \beta_{n, i} J_{E_{2}}^{p}\left(I-\Pi_{A K}^{p} B_{\delta_{n}}^{T_{i}}\right) A u_{n, i}\right\|^{p-1}},
$$


with, $\lim _{n \rightarrow \infty} \eta_{n, 0}=0, \eta_{n, 0} \leq \sum_{i=1}^{\infty} \eta_{n, i}$, for $M \geq 0, \eta_{n-1,0} \leq \sum_{i=1}^{\infty} \eta_{n-1, i} \leq \sum_{n=1}^{\infty} \sum_{i=1}^{\infty} \eta_{n-1, i} M<\infty$, $\sum_{i=0}^{\infty} \eta_{n, i}=\sum_{i=0}^{\infty} \alpha_{n, i}=\sum_{i=0}^{\infty} \beta_{n, i}=1$ and $k_{n}=\max _{i \in I}\left\{k_{n, i}\right\}$. If $\Gamma=\Omega \cap \omega \cap \Im \neq \varnothing$, then $\left\{x_{n}\right\}$ converges strongly to $x^{*} \in \Gamma$, where $\sum_{i=0}^{\infty} \beta_{n, i} \Pi_{A K}^{p} B_{\delta_{n}}^{T_{i}}\left(x^{*}\right)=\sum_{i=0}^{\infty} \beta_{n, i} B_{\delta_{n}}^{T_{i}}\left(x^{*}\right)$, for each $i \in I$.

Proof. For $x, y \in K$ and $i \in I$, let $\Phi_{i}(x, y)=g_{i}(x, y)+\left\langle J_{E_{1}}^{p} C_{i} x, y-x\right\rangle+\theta_{i}(y)-\theta_{i}(x)$. Since $g_{i}$ are bi-functions satisfying $(A 1)-(A 4)$ in (3) and $C_{i}$ are monotone and Lipschitz continuous mappings, and $\theta_{i}$ are convex and lower semicontinuous functions, therefore $\Phi_{i}(i \in I)$ satisfy the conditions $(A 1)-(A 4)$ in (3), and hence the algorithm (29) can be written as follows:

$$
\left\{\begin{array}{l}
\Phi_{i}\left(u_{n, i}, y\right)+\left\langle J_{E_{1}}^{p} G_{n, i} x_{n}, y-u_{n, i}\right\rangle+\frac{1}{r_{n, i}}\left\langle y-u_{n, i}, J_{E_{1}}^{p} u_{n, i}-J_{E_{1}}^{p} x_{n}\right\rangle \geq 0 \\
\forall y \in K, \forall i \in I, \\
y_{n}=J_{E_{1}^{*}}^{q}\left(\sum_{i=0}^{\infty} \alpha_{n, i} B_{\delta_{n}}^{U_{i}}\left(J_{E_{1}}^{p} u_{n, i}-\sum_{i=0}^{\infty} \beta_{n, i} \lambda_{n} A^{*} J_{E_{2}}^{p}\left(I-\Pi_{A K}^{p} B_{\delta_{n}}^{T_{i}}\right) A u_{n, i}\right)\right), \\
x_{n+1}=J_{E_{1}^{*}}^{q}\left(\eta_{n, 0} J_{E_{1}}^{p}\left(f\left(x_{n}\right)\right)+\sum_{i=1}^{\infty} \eta_{n, i} J_{E_{1}}^{p}\left(S_{n, i}\left(y_{n}\right)\right)\right) n \geq 1 .
\end{array}\right.
$$

We will divide the proof into four steps.

Step One: We show that $\left\{x_{n}\right\}$ is a bounded sequence.

Assume that $\left\|\sum_{i=0}^{\infty} \beta_{n, i} J_{E_{2}}^{p}\left(I-\Pi_{A K}^{p} B_{\delta_{n}}^{T_{i}}\right) A u_{n, i}\right\|=0$ and $\left\|J_{E_{1}}^{p} G_{n, i} x_{n}\right\|=0$. Then, by (32), we have

$$
\Phi_{i}\left(u_{n, i}, y\right)+\frac{1}{r_{n, i}}\left\langle y-u_{n, i}, J_{E_{1}}^{p} u_{n, i}-J_{E_{1}}^{p} x_{n}\right\rangle \geq 0 \forall y \in K, \forall i \in I .
$$

By (33) and Lemma 13, for each $i \in I$, we have that $u_{n, i}=J_{E_{1}^{*}}^{q}\left(Y_{r_{n, i}}\left(J_{E_{1}}^{p} x_{n}\right)\right)$. By Lemma 4 and for $v \in \Gamma$ and $v=\mathrm{Y}_{r_{n, i}} v$, we have

$$
\triangle_{p}\left(u_{n, i}, v\right)=V_{p}\left(Y_{r_{n, i}}\left(J_{E_{1}}^{p} x_{n}\right), v\right) \leq V_{p}\left(J_{E_{1}}^{p} x_{n}, v\right)=\triangle_{p}\left(x_{n}, v\right) .
$$

In addition, for each $i \in I$, let $v=B_{\gamma}^{U_{i}} v$. By Lemma 4 and for $v \in \Gamma$, we have

$$
\triangle_{p}\left(y_{n}, v\right)=V_{p}\left(\sum_{i=0}^{\infty} \alpha_{n, i} B_{\delta_{n}}^{U_{i}} J_{E_{1}}^{p} u_{n, i}, v\right) \leq \triangle_{p}\left(u_{n, i}, v\right)
$$
have that

Now assume that $\left\|\sum_{i=0}^{\infty} \beta_{n, i} j_{E_{2}}^{p}\left(I-\Pi_{A K}^{p} B_{\delta_{n}}^{T_{i}}\right) A u_{n, i}\right\| \neq 0$ and $\left\|J_{E_{1}}^{p} G_{n, i} x_{n}\right\| \neq 0$. Then by (32), we $\Phi_{i}\left(u_{n, i}, y\right)+\frac{1}{r_{n, i}}\left\langle y-u_{n, i}, J_{E_{1}}^{p} u_{n, i}-\left(J_{E_{1}}^{p} x_{n}-r_{n, i} J_{E_{1}}^{p} G_{n, i} x_{n}\right)\right\rangle \geq 0 \forall y \in K, \forall i \in I$.

By (36) and Lemma 13, for each $i \in I$, we have $u_{n, i}=J_{E_{1}^{*}}^{q}\left(Y_{r_{n, i}}\left(J_{E_{1}}^{p} x_{n}-r_{n, i} J_{E_{1}}^{p} G_{n, i} x_{n}\right)\right)$. For $v \in \Gamma$, by (22) in Lemma 15, we get

$$
\triangle_{p}\left(u_{n, i}, v\right) \leq \triangle_{p}\left(x_{n}, v\right) .
$$

In addition, for each $i \in I, v \in \Gamma$, (21) in Lemma 15 gives

$$
\triangle_{p}\left(y_{n}, v\right) \leq \triangle_{p}\left(u_{n, i}, v\right) .
$$


Let $u_{n, i}=0$. By Lemma 1 , we have

$$
\triangle_{p}\left(u_{n, i}, v\right)=\frac{1}{p}\|v\|^{p}
$$

and by (27), (39), Lemmas 4 and 15, we have

$$
\begin{aligned}
& \triangle_{p}\left(y_{n}, v\right) \leq \frac{1}{q}\left\|\sum_{i=0}^{\infty} \beta_{n, i} \lambda_{n} A^{*} J_{E_{2}}^{p}\left(I-\Pi_{A K}^{p} B_{\delta_{n}}^{T_{i}}\right) A u_{n, i}\right\|^{p} \\
& +\triangle_{p}\left(u_{n, i}, v\right)+\lambda_{n}\left\langle\sum_{i=0}^{\infty} \beta_{n, i} J_{E_{2}}^{p}\left(I-\Pi_{A K}^{p} B_{\delta_{n}}^{T_{i}}\right) A u_{n, i}, A u_{n, i}\right\rangle \\
& \quad-\lambda_{n}\left\langle\sum_{i=0}^{\infty} \beta_{n, i} J_{E_{2}}^{p}\left(I-\Pi_{A K}^{p} B_{\delta_{n}}^{T_{i}}\right) A u_{n, i} \sum_{i=0}^{\infty} \beta_{n, i}\left(I-\Pi_{A K}^{p} B_{\delta_{n}}^{T_{i}}\right) A u_{n, i}\right\rangle .
\end{aligned}
$$

However, by (30) and (40), we have

$$
\begin{aligned}
& \triangle_{p}\left(y_{n}, v\right) \\
& \leq \frac{1}{q} \frac{1}{\|A\|^{p}} \frac{\left\langle\sum_{i=0}^{\infty} \beta_{n, i} J_{E_{2}}^{p}\left(I-\Pi_{A K}^{p} B_{\delta_{n}}^{T_{i}}\right) A u_{n, i}, \sum_{i=0}^{\infty} \beta_{n, i}\left(I-\Pi_{A K}^{p} B_{\delta_{n}}^{T_{i}}\right) A u_{n, i}\right\rangle^{p}}{\left\|\sum_{i=0}^{\infty} \beta_{n, i} J_{E_{2}}^{p}\left(I-\Pi_{A K}^{p} B_{\delta_{n}}^{T_{i}}\right) A u_{n, i}\right\|^{p}} \\
& +\triangle_{p}\left(u_{n, i}, v\right)+\lambda_{n}\left\langle\sum_{i=0}^{\infty} \beta_{n, i} J_{E_{2}}^{p}\left(I-\Pi_{A K}^{p} B_{\delta_{n}}^{T_{i}}\right) A u_{n, i}, A u_{n, i}\right\rangle \\
& -\lambda_{n}\left\langle\sum_{i=0}^{\infty} \beta_{n, i} J_{E_{2}}^{p}\left(I-\Pi_{A K}^{p} B_{\delta_{n}}^{T_{i}}\right) A u_{n, i} \sum_{i=0}^{\infty} \beta_{n, i}\left(I-\Pi_{A K}^{p} B_{\delta_{n}}^{T_{i}}\right) A u_{n, i}\right\rangle \\
& \leq \triangle_{p}\left(u_{n, i}, v\right) \\
& -\frac{1}{\|A\|^{p}} \frac{\left\langle\sum_{i=0}^{\infty} \beta_{n, i} J_{E_{2}}^{p}\left(I-\Pi_{A K}^{p} B_{\delta_{n}}^{T_{i}}\right) A u_{n, i}, \sum_{i=0}^{\infty} \beta_{n, i}\left(I-\Pi_{A K}^{p} B_{\delta_{n}}^{T_{i}}\right) A u_{n, i}\right\rangle^{p}}{\left\|\sum_{i=0}^{\infty} \beta_{n, i} J_{E_{2}}^{p}\left(I-\Pi_{A K}^{p} B_{\delta_{n}}^{T_{i}}\right) A u_{n, i}\right\|^{p}}
\end{aligned}
$$

This implies that

$$
\triangle_{p}\left(y_{n}, v\right) \leq \triangle_{p}\left(u_{n, i}, v\right)
$$

By (42) and (37), we get

$$
\triangle_{p}\left(y_{n}, v\right) \leq \triangle_{p}\left(x_{n}, v\right)
$$

In addition, it follows from the assumption $\eta_{n, 0} \leq \sum_{i=1}^{\infty} \eta_{n, i},(43)$, Definition 3, Lemmas 9 and 4 


$$
\begin{aligned}
\triangle_{p} & \left(x_{n+1}, v\right) \\
& =\triangle_{p}\left(J_{E_{1}^{*}}^{q}\left(\eta_{n, 0} J_{E_{1}}^{p}\left(f\left(x_{n}\right)\right)+\sum_{i=1}^{\infty} \eta_{n, i} J_{E_{1}}^{p}\left(S_{n, i}\left(y_{n}\right)\right)\right), v\right) \\
& =V_{p}\left(\eta_{n, 0} J_{E_{1}}^{p}\left(f\left(x_{n}\right)\right)+\sum_{i=1}^{\infty} \eta_{n, i} J_{E_{1}}^{p}\left(S_{n, i}\left(y_{n}\right)\right), v\right) \\
& \leq \eta_{n, 0} V_{p}\left(J_{E_{1}}^{p}\left(f\left(x_{n}\right)\right), v\right)+\sum_{i=1}^{\infty} \eta_{n, i} V_{p}\left(J_{E_{1}}^{p}\left(S_{n, i}\left(y_{n}\right)\right), v\right) \\
& \leq \eta_{n, 0} \zeta \triangle_{p}\left(x_{n}, v\right)+\eta_{n, 0}\left(\triangle_{p}(f(v), v)\right. \\
& \left.+\left\langle J_{E_{1}}^{p} x_{n}-J_{E_{1}}^{p} f(v), f(v)-v\right\rangle\right)+\sum_{i=1}^{\infty} \eta_{n, i} k_{n, i} \triangle_{p}\left(y_{n}, v\right) \\
& \leq \eta_{n, 0}\left(\triangle_{p}(f(v), v)+\left\langle J_{E_{1}}^{p} x_{n}-J_{E_{1}}^{p} f(v), f(v)-v\right\rangle\right) \\
& +\left(\eta_{n, 0} \zeta+\sum_{i=1}^{\infty} \eta_{n, i} k_{n, i}\right) \triangle_{p}\left(x_{n}, v\right) \\
& \leq \eta_{n, 0}\left(\triangle_{p}(f(v), v)+\left\langle J_{E_{1}}^{p} x_{n}-J_{E_{1}}^{p} f(v), f(v)-v\right\rangle\right) \\
& +\left(\sum_{i=1}^{\infty} \eta_{n, i}\left(\zeta+k_{n, i}\right)\right) \triangle_{p}\left(x_{n}, v\right) \\
& \leq \max \left\{\frac{\left(\triangle_{p}(f(v), v)+\left\langle J_{E_{1}}^{p} x_{1}-J_{E_{1}}^{p} f(v), f(v)-v\right\rangle\right)}{\zeta+k_{1, i}}, \triangle_{p}\left(x_{1}, v\right)\right\} .
\end{aligned}
$$

By (44), we conclude that $\left\{x_{n}\right\}$ is bounded, and hence, from (42), (34), (35), (44), (38), and (37), $\left\{y_{n}\right\}$ and $\left\{u_{n, i}\right\}$ are also bounded.

Step Two: We show that $\lim _{m \rightarrow \infty} \triangle_{p}\left(x_{n+1}, x_{n}\right)=0$. By Lemmas 1, 4, 10, and 7, we have, by the convexity of $\triangle_{p}$ in the first argument and for $\eta_{n-1,0} \leq \sum_{i=1}^{\infty} \eta_{n-1, i}$

$$
\begin{aligned}
& \triangle_{p}\left(x_{n+1}, x_{n}\right)=\triangle_{p}\left(J_{E_{1}^{*}}^{q}\left(\eta_{n, 0} J_{E_{1}}^{p}\left(f\left(x_{n}\right)\right)+\sum_{i=1}^{\infty} \eta_{n, i} J_{E_{1}}^{p}\left(S_{n, i}\left(y_{n}\right)\right)\right),\right. \\
& \left.\quad J_{E_{1}^{*}}^{q}\left(\eta_{n-1,0} J_{E_{1}}^{p}\left(f\left(x_{n-1}\right)\right)+\sum_{i=1}^{\infty} \eta_{n-1, i} J_{E_{1}}^{p}\left(S_{n-1, i}\left(y_{n-1}\right)\right)\right)\right) \\
& \quad \leq \eta_{n, 0} \triangle_{q}^{*}\left(J_{E_{1}}^{p}\left(f\left(x_{n}\right)\right), \eta_{n-1,0} J_{E_{1}}^{p}\left(f\left(x_{n-1}\right)\right)+\sum_{i=1}^{\infty} \eta_{n-1, i} J_{E_{1}}^{p}\left(S_{n-1, i}\left(y_{n-1}\right)\right)\right) \\
& \quad+\sum_{i=1}^{\infty} \eta_{n, i} \triangle_{q}^{*}\left(J_{E_{1}}^{p}\left(S_{n, i}\left(y_{n}\right)\right), \eta_{n-1,0} J_{E_{1}}^{p}\left(f\left(x_{n-1}\right)\right)+\sum_{i=1}^{\infty} \eta_{n-1, i} J_{E_{1}}^{p}\left(S_{n-1, i}\left(y_{n-1}\right)\right)\right) \\
& \quad \leq \eta_{n, 0}\left(\triangle_{q}^{*}\left(J_{E_{1}}^{p}\left(f\left(x_{n}\right), J_{E_{1}}^{p}\left(f\left(x_{n-1}\right)\right)\right)\right)\right. \\
& \quad+\sum_{i=1}^{\infty} \eta_{n-1, i}\left(\sum_{i=1}^{\infty} \eta_{n, i} \frac{1}{p}\left\|S_{n-1, i}\left(y_{n-1}\right)\right\|^{p}+\eta_{n, 0}\left\|f\left(x_{n}\right)\right\|\left\|J_{E_{1}}^{p}\left(S_{n-1, i}\left(y_{n-1}\right)\right)\right\|\right) \\
& \quad+\eta_{n-1,0}\left(\eta_{n, 0} \frac{1}{p}\left\|f\left(x_{n-1}\right)\right\|^{p}+\sum_{i=1}^{\infty} \eta_{n, i}\left\|S_{n, i}\left(y_{n}\right)\right\|\left\|J_{E_{1}}^{p}\left(f\left(x_{n-1}\right)\right)\right\|\right) \\
& \quad+\sum_{i=1}^{\infty} \eta_{n, i} \triangle_{q}^{*}\left(\left(J_{E_{1}}^{p} S_{n, i}\left(y_{n}\right), J_{E_{1}}^{p} S_{n-1, i}\left(y_{n-1}\right)\right)\right. \\
& \quad \leq\left(1-\eta_{n, 0}(1-\zeta)\right) \triangle_{p}\left(x_{n}, x_{n-1}\right)+\sum_{i=1}^{\infty} \eta_{n, i} \sup _{n, n-1 \geq 1}\left\{\triangle_{p}\left(S_{n, i}\left(y_{n}\right), S_{n-1, i}\left(y_{n-1}\right)\right)\right\} \\
& \quad+\sum_{i=1}^{\infty} \eta_{n-1, i} M,
\end{aligned}
$$


where

$$
\left.M=\max \left\{\max \left\{\| f\left(x_{n}\right)\right)\|,\| S_{n-1, i}\left(y_{n-1}\right) \|\right\}, \max \left\{\left\|f\left(x_{n-1}\right)\right\|,\left\|S_{n, i}\left(y_{n}\right)\right\|\right\}\right\} .
$$

In view of the assumption $\sum_{n=1}^{\infty} \sum_{i=1}^{\infty} \eta_{n-1, i} M<\infty$ and (45), Lemmas 11 and 8 imply

$$
\lim _{n \rightarrow \infty} \triangle_{p}\left(x_{n+1}, x_{n}\right)=0 .
$$

Step Three: We show that $\lim _{n \rightarrow \infty} \triangle_{p}\left(S_{n, i} y_{n}, y_{n}\right)=0$.

For each $i \in I$, we have

$$
\triangle_{p}\left(S_{i}\left(y_{n}\right), v\right) \leq \triangle_{p}\left(y_{n}, v\right)
$$

Then,

$$
\begin{aligned}
0 & \leq \triangle_{p}\left(y_{n}, v\right)-\triangle_{p}\left(S_{i}\left(y_{n}\right), v\right) \\
& =\triangle_{p}\left(y_{n}, v\right)-\triangle_{p}\left(x_{n+1}, v\right)+\triangle_{p}\left(x_{n+1}, v\right)-\triangle_{p}\left(S_{i}\left(y_{n}\right), v\right) \\
& \leq \triangle_{p}\left(x_{n}, v\right)-\triangle_{p}\left(x_{n+1}, v\right)+\triangle_{p}\left(x_{n+1}, v\right)-\triangle_{p}\left(S_{i}\left(y_{n}\right), v\right) \\
& =\triangle_{p}\left(x_{n}, v\right)-\triangle_{p}\left(x_{n+1}, v\right)+\triangle_{p}\left(J_{E_{1}^{*}}^{q}\left(\eta_{n, 0} J_{E_{1}}^{p}\left(f\left(x_{n}\right)\right)+\sum_{i=1}^{\infty} \eta_{n, i} J_{E_{1}}^{p}\left(S_{i}\left(y_{n}\right)\right)\right), v\right) \\
& -\triangle_{p}\left(S_{i}\left(y_{n}\right), v\right) \\
& \leq \triangle_{p}\left(x_{n}, v\right)-\triangle_{p}\left(x_{n+1}, v\right)+\eta_{n, 0} \triangle_{p}\left(f\left(x_{n}\right), v\right)-\eta_{n, 0} \triangle_{p}\left(S_{i}\left(y_{n}\right), v\right) \\
& \longrightarrow 0 \text { as } n \rightarrow \infty .
\end{aligned}
$$

By (47) and Definition 2, we get

$$
\lim _{n \rightarrow \infty} \triangle_{p}\left(S_{i} y_{n}, y_{n}\right)=0
$$

By uniform continuity of $S$, we have

$$
\lim _{n \rightarrow \infty} \triangle_{p}\left(S_{n, i} y_{n}, y_{n}\right)=0 .
$$

Step Four: We show that $x_{n} \rightarrow x^{*} \in \Gamma$.

Note that,

$$
\begin{aligned}
& \triangle_{p}\left(x_{n+1}, y_{n}\right)=\triangle_{p}\left(J_{E_{1}^{*}}^{q}\left(\eta_{n, 0} J_{E_{1}}^{p}\left(f\left(x_{n}\right)\right)+\sum_{i=1}^{\infty} \eta_{n, i} J_{E_{1}}^{p}\left(S_{n, i}\left(y_{n}\right)\right)\right), y_{n}\right) \\
& \leq \eta_{n, 0} \triangle_{p}\left(f\left(x_{n}\right), y_{n}\right)+\sum_{i=1}^{\infty} \eta_{n, i} \triangle_{p}\left(S_{n, i}\left(y_{n}\right), y_{n}\right) \\
& \leq \eta_{n, 0}\left(\zeta \triangle_{p}\left(x_{n}, y_{n}\right)+\triangle_{p}\left(f\left(y_{n}\right), y_{n}\right)+\left\langle f\left(x_{n}\right)-f\left(y_{n}\right), J_{E_{1}}^{p} f\left(y_{n}\right)-J_{E_{1}}^{p} y_{n}\right\rangle\right) \\
& +\sum_{i=1}^{\infty} \eta_{n, i} \triangle_{p}\left(S_{n, i}\left(y_{n}\right), y_{n}\right) \\
& \leq\left(1-\eta_{n, 0}(1-\zeta)\right) \triangle_{p}\left(x_{n}, y_{n}\right) \\
& +\eta_{n, 0}\left(\triangle_{p}\left(f\left(y_{n}\right), y_{n}\right)+\left\langle f\left(x_{n}\right)-f\left(y_{n}\right), J_{E_{1}}^{p} f\left(y_{n}\right)-J_{E_{1}}^{p} y_{n}\right\rangle\right) \\
& +\sum_{i=1}^{\infty} \eta_{n, i} \triangle_{p}\left(S_{n, i}\left(y_{n}\right), y_{n}\right) .
\end{aligned}
$$


By (49), (50), and Lemma 8, we have

$$
\lim _{n \rightarrow \infty} \triangle_{p}\left(x_{n}, y_{n}\right)=0 .
$$

Therefore, by (51) and the boundedness of $\left\{y_{n}\right\}$, and since by (46), $\left\{x_{n}\right\}$ is Cauchy, we can assume without loss of generality that $y_{n} \rightarrow x^{*}$ for some $x^{*} \in E_{1}$. It follows from Lemmas 2, 3, and (48) that $x^{*}=S_{i} x^{*}$, for each $i \in I$. This means that $x^{*} \in \Im$.

In addition, by (31) and the fact that $u_{n, i} \rightarrow x^{*}$ as $n \rightarrow \infty$, we arrive at

$$
\frac{\left(J_{E_{1}}^{p} u_{n, i}-J_{E_{1}}^{p} y_{n}\right)-\sum_{i=0}^{\infty} \beta_{n, i} \lambda_{n} A^{*} J_{E_{2}}^{p}\left(I-\Pi_{A K}^{p} B_{\delta_{n}}^{T_{i}}\right) A u_{n, i}}{\delta_{n}} \in \sum_{i=0}^{\infty} \alpha_{n, i} U_{i}\left(y_{n}\right) .
$$

By (21), we have

$$
\left\|\sum_{i=0}^{\infty} \beta_{n, i}\left(I-\Pi_{A K}^{p} B_{\delta_{n}}^{T_{i}}\right) A u_{n, i}\right\| \leq\left[\frac{\triangle_{p}\left(u_{n, i}, v\right)-\triangle_{p}\left(y_{n}, v\right)}{\|A\|^{-1}[1-l]}\right] \longrightarrow 0 \text { as } n \rightarrow \infty,
$$

and by (41), we have

$$
\left\|\sum_{i=0}^{\infty} \beta_{n, i}\left(I-\Pi_{A K}^{p} B_{\delta_{n}}^{T_{i}}\right) A u_{n, i}\right\| \leq\left[\frac{\triangle_{p}\left(u_{n, i}, v\right)-\triangle_{p}\left(y_{n}, v\right)}{(p\|A\|)^{-1}}\right]^{\frac{1}{p}} \longrightarrow 0 \text { as } n \rightarrow \infty .
$$

From (53), (54), and (52), by passing $n$ to infinity in (52), we have that $0 \in \sum_{i=0}^{\infty} \alpha_{n, i} U_{i}\left(x^{*}\right)$. This implies that $x^{*} \in \operatorname{SOLVIP}\left(U_{i}\right)$. In addition, by (48), we have $A y_{n} \rightarrow A x^{*}$. Thus, by (53), (54) and an application of the demi-closeness of $\sum_{i=0}^{\infty} \beta_{n, i}\left(I-\Pi_{A K}^{p} B_{\delta_{n}}^{T_{i}}\right)$ at zero, we have that $0 \in \sum_{i=0}^{\infty} \beta_{n, i} T_{i}\left(A x^{*}\right)$. Therefore, $A x \in \operatorname{SOLVIP}\left(T_{i}\right)$ as $\sum_{i=0}^{\infty} \beta_{n, i} \Pi_{A K}^{p} B_{\delta}^{T_{i}}\left(A x^{*}\right)=\sum_{i=0}^{\infty} \beta_{n, i} B_{\delta}^{T_{i}}\left(A x^{*}\right)$. This means that $x^{*} \in \Omega$.

Now, we show that $x^{*} \in\left(\cap_{i=1}^{\infty} G M E P\left(\theta_{i}, C_{i}, G_{i}, g_{i}\right)\right.$. By (32), we have

$$
\begin{array}{r}
\Phi_{i}\left(u_{n, i}, y\right)+\left\langle J_{E_{1}}^{p} G_{n, i} x_{n}, y-u_{n, i}\right\rangle+\frac{1}{r_{n, i}}\left\langle y-u_{n, i}, J_{E_{1}}^{p} u_{n, i}-J_{E_{1}}^{p} x_{n}\right\rangle \geq 0 \\
\forall y \in K, \forall i \in I,
\end{array}
$$

Since $\Phi_{i}$, for each $i \in I$, are monotone, that is, for all $y \in K$,

$$
\begin{aligned}
& \Phi_{i}\left(u_{n, i}, y\right)+\Phi_{i}\left(y, u_{n, i}\right) \leq 0 \\
& \Rightarrow \frac{1}{r_{n, i}}\left\langle y-u_{n, i}, J_{E_{1}}^{p} u_{n, i}-J_{E_{1}}^{p} x_{n}\right\rangle \\
& \geq \Phi_{i}\left(y, u_{n, i}\right)+\left\langle J_{E_{1}}^{p} G_{n, i} x_{n}, y-u_{n, i}\right\rangle,
\end{aligned}
$$

therefore,

$$
\frac{1}{r_{n, i}}\left\langle y-u_{n, i}, J_{E_{1}}^{p} u_{n, i}-J_{E_{1}}^{p} x_{n}\right\rangle \geq \Phi_{i}\left(y, u_{n, i}\right)+\left\langle J_{E_{1}}^{p} G_{n, i} x_{n}, y-u_{n, i}\right\rangle .
$$

By the lower semicontinuity of $\Phi_{i}$, for each $i \in I$, the weak upper semicontinuity of $G$, and the facts that, for each $i \in I, u_{n, i} \rightarrow x^{*}$ as $n \rightarrow \infty$ and $J^{p}$ is norm - to - weak* uniformly continuous on a bounded subset of $E_{1}$, we have

$$
0 \geq \Phi_{i}\left(y, x^{*}\right)+\left\langle J_{E_{1}}^{p} G_{n, i} x^{*}, y-x^{*}\right\rangle .
$$


Now, we set $y_{t}=t y+(1-t) x^{*} \in K$. From (55), we get

$$
0 \geq \Phi_{i}\left(y_{t}, x^{*}\right)+\left\langle J_{E_{1}}^{p} G_{n, i} x^{*}, y_{t}-x^{*}\right\rangle .
$$

From (56), and by the convexity of $\Phi_{i}$, for each $i \in I$, in the second variable, we arrive at

$$
\begin{aligned}
0 & =\Phi_{i}\left(y_{t}, y_{t}\right) \leq t \Phi_{i}\left(y_{t}, y\right)+(1-t) \Phi_{i}\left(y_{t}, x^{*}\right) \\
& \leq t \Phi_{i}\left(y_{t}, y\right)+(1-t)\left\langle J_{E_{1}}^{p} G_{n, i} x^{*}, y_{t}-x^{*}\right\rangle \\
& \leq t \Phi_{i}\left(y_{t}, y\right)+(1-t) t\left\langle J_{E_{1}}^{p} G_{n, i} x^{*}, y-x^{*}\right\rangle,
\end{aligned}
$$

which implies that

$$
\Phi_{i}\left(y_{t}, y\right)+(1-t)\left\langle J_{E_{1}}^{p} G_{n, i} x^{*}, y-x^{*}\right\rangle \geq 0 .
$$

From (57), by the lower semicontinuity of $\Phi_{i}$, for each $i \in I$, we have for $y_{t} \rightarrow x^{*}$ as $t \rightarrow 0$

$$
\Phi_{i}\left(x^{*}, y\right)+\left\langle J_{E_{1}}^{p} G_{n, i} x^{*}, y-x^{*}\right\rangle \geq 0 .
$$

Therefore, by (58) we can conclude that $x^{*} \in\left(\cap_{i=1}^{\infty} \operatorname{GMEP}\left(\theta_{i}, C_{i}, G_{i}, g_{i}\right)\right.$. This means that $x^{*} \in \omega$. Hence, $x^{*} \in \Gamma$.

Finally, we show that $x_{n} \rightarrow x^{*}$, as $n \rightarrow \infty$. By Definition 3, we have

$$
\begin{aligned}
& \triangle_{p}\left(x_{n+1}, x^{*}\right) \\
& =\triangle_{p}\left(J_{E_{1}^{*}}^{q}\left(\eta_{n, 0} J_{E_{1}}^{p}\left(f\left(x_{n}\right)\right)+\sum_{i=1}^{\infty} \eta_{n, i} J_{E_{1}}^{p}\left(G_{n, i}\left(y_{n}\right)\right)\right), x^{*}\right) \\
& \leq \eta_{n, 0} \triangle_{q}^{*}\left(J_{E_{1}}^{p}\left(f\left(u_{n}\right)\right), J_{E_{1}}^{p} x^{*}\right)+\sum_{i=1}^{\infty} \eta_{n, i} \triangle_{q}^{*}\left(J_{E_{1}}^{p}\left(G_{n, i}\left(y_{n}\right)\right), J_{E_{1}}^{p} x^{*}\right) \\
& \leq \eta_{n, 0} \zeta \triangle_{p}\left(x_{n}, x^{*}\right)+\eta_{n, 0}\left(\triangle_{p}\left(f\left(x^{*}\right), x^{*}\right)\right. \\
& \left.+\left\langle J_{E_{1}}^{p} x_{n}-J_{E_{1}}^{p} f\left(x^{*}\right), f\left(x^{*}\right)-x^{*}\right\rangle\right)+\sum_{i=1}^{\infty} \eta_{n, i} k_{n} \triangle_{p}\left(y_{n}, x^{*}\right) \\
& \leq \eta_{n, 0}\left(\triangle_{p}\left(f\left(x^{*}\right), x^{*}\right)+\left\langle J_{E_{1}}^{p} x_{n}-J_{E_{1}}^{p} f\left(x^{*}\right), f\left(x^{*}\right)-x^{*}\right\rangle\right) \\
& +\left(1-\sum_{i=1}^{\infty} \eta_{n, i}\left(1-k_{n}\right)\right) \triangle_{p}\left(x_{n}, x^{*}\right) .
\end{aligned}
$$

By (59) and Lemma 8, we have that

$$
\lim _{n \rightarrow \infty} \triangle_{p}\left(x_{n}, x^{*}\right)=0
$$

The proof is completed.

In Theorem $1, i=0$ leads to the following new result.

Corollary 1. Let $g: K \times K \rightarrow R$ be bifunctions satisfying $(A 1)-(A 4)$ in (3). Let $\left(I-\Pi_{A K}^{p} B_{\delta}^{T}\right)$ be demiclosed at zero. Suppose that $x_{1} \in E_{1}$ is chosen arbitrarily and the sequence $\left\{x_{n}\right\}$ is defined as follows:

$$
\left\{\begin{array}{l}
g\left(u_{n}, y\right)+\left\langle J_{E_{1}}^{p} C u_{n}+J_{E_{1}}^{p} G_{n} x_{n}, y-u_{n}\right\rangle+\theta(y)-\theta\left(u_{n}\right) \\
+\frac{1}{r_{n}}\left\langle y-u_{n}, J_{E_{1}}^{p} u_{n}-J_{E_{1}}^{p} x_{n}\right\rangle \geq 0 \forall y \in K, \\
y_{n}=J_{E_{1}^{*}}^{q}\left(B_{\delta_{n}}^{U}\left(J_{E_{1}}^{p} u_{n}-\lambda_{n} A^{*} J_{E_{2}}^{p}\left(I-\Pi_{A K}^{p} B_{\delta_{n}}^{T}\right) A u_{n}\right)\right), \\
x_{n+1}=J_{E_{1}^{*}}^{q}\left(\eta_{n} J_{E_{1}}^{p}\left(f\left(x_{n}\right)\right)+\left(1-\eta_{n}\right) J_{E_{1}}^{p}\left(S_{n}\left(y_{n}\right)\right)\right) n \geq 1,
\end{array}\right.
$$


where $r_{n}=\frac{1}{\left\|J_{E_{1}}^{p} G_{n} x_{n}\right\|^{\prime}}, \mu_{n}=\frac{1}{\left\|x_{n}\right\|^{p-1}}$ and $\gamma \in(0,1)$ such that $\rho_{E_{1}^{*}}\left(\mu_{n}\right)=\frac{\gamma\left\langle J_{E_{1}}^{p} G_{n} x_{n}, x_{n}-v\right\rangle}{{ }^{q} G_{q}\left\|x_{n}\right\|^{p}\left\|J_{E_{1}}^{p} G_{n} x_{n}\right\|}$, and

$$
\lambda_{n}=\left\{\begin{array}{l}
\frac{1}{\|A\|} \frac{1}{\left\|J_{E_{2}}^{p}\left(I-\Pi_{A K}^{p} B_{\delta_{n}}^{T}\right) A u_{n}\right\|^{\prime}}, u_{n} \neq 0 \\
\frac{1}{\|A\|^{p}} \frac{\left\|J_{E_{2}}^{p}\left(I-\Pi_{A K}^{p} B_{\delta_{n}}^{T}\right) A u_{n}\right\|^{p(p-1)}}{\left\|J_{E_{2}}^{p}\left(I-\Pi_{A K}^{p} B_{\delta_{n}}^{T}\right) A u_{n}\right\|^{p}}, u_{n}=0
\end{array}\right.
$$

and $\iota \in(0,1)$ and $\tau_{n}=\frac{1}{\left\|u_{n}\right\|^{p-1}}$ are chosen such that

$$
\rho_{E_{1}^{*}}\left(\tau_{n}\right)=\frac{\iota}{2^{q} G_{q}\|A\|} \times \frac{\left\|J_{E_{2}}^{p}\left(I-\Pi_{A K}^{p} B_{\delta_{n}}^{T}\right) A u_{n}\right\|^{p}}{\left\|u_{n}\right\|^{p}\left\|J_{E_{2}}^{p}\left(I-\Pi_{A K}^{p} B_{\delta_{n}}^{T}\right) A u_{n}\right\|^{p-1}},
$$

and $\lim _{n \rightarrow \infty} \eta_{n}=0$, for $M \geq 0, \sum_{n=1}^{\infty} \eta_{n-1} M<\infty$, and $\eta_{n} \leq \frac{1}{2}$. If $\Gamma=\Omega \cap \omega \cap \Im \neq \varnothing$, then $\left\{x_{n}\right\}$ converges strongly to $x^{*} \in \Gamma$, where $\Pi_{A K}^{p} B_{\delta_{n}}^{T}\left(x^{*}\right)=B_{\delta_{n}}^{T}\left(x^{*}\right)$.

\section{Application to Generalized Mixed Equilibrium Problem, Split Hammerstein Integral Equations and Fixed Point Problem}

Definition 4. Let $C \subset \mathbb{R}^{n}$ be bounded. Let $k: C \times C \rightarrow \mathbb{R}$ and $f: C \times \mathbb{R} \rightarrow \mathbb{R}$ be measurable real-valued functions. An integral equation of Hammerstien-type has the form

$$
u(x)+\int_{C} k(x, y) f(y, u(y)) d y=w(x),
$$

where the unknown function $u$ and non-homogeneous function $w$ lies in a Banach space $E$ of measurable real-valued functions. By transforming the above equation, we have that

$$
u+K F u=w,
$$

and therefore, without loss of generality, we have

$$
u+K F u=0 .
$$
such that

The split Hammerstein integral equations problem is formulated as finding $x^{*} \in E_{1}$ and $y^{*} \in E_{1}^{*}$

$$
x^{*}+K F x^{*}=0 \text { with } F x^{*}=y^{*} \text { and } K y^{*}+x^{*}=0
$$

and $A x^{*} \in E_{2}$ and $A y^{*} \in E_{2}^{*}$ such that

$$
A x^{*}+K^{\prime} F^{\prime} A x^{*}=0 \text { with } F^{\prime} A x^{*}=A y^{*} \text { and } K^{\prime} A y^{*}+A x^{*}=0
$$

where $F: E_{1} \rightarrow E_{1}^{*}, K: E_{1}^{*} \rightarrow E_{1}$ and $F^{\prime}: E_{2} \rightarrow E_{2}^{*}, K^{\prime}: E_{2}^{*} \rightarrow E_{2}$ are maximal monotone mappings.

Lemma 16 ([21]). Let $E$ be a Banach space. Let $F: E \rightarrow E^{*}, K: E^{*} \rightarrow E$ be bounded and maximal monotone operators. Let $D: E \times E^{*} \rightarrow E^{*} \times E$ be defined by $D(x, y)=(F x-y, K y+x)$ for all $(x, y) \in E \times E^{*}$. Then, the mapping $D$ is maximal monotone. 
By Lemma 16, if $K, K^{\prime}$, and $F, F^{\prime}$ are multi-valued maximal monotone operators then, we have two resolvent mappings,

$$
B_{\delta}^{D}=\left(J_{E_{1}}^{p}+\delta J_{E_{1}}^{p} D\right)^{-1} J_{E_{1}}^{p} \text { and } B_{\delta}^{D^{\prime}}=\left(J_{E_{2}}^{p}+\delta J_{E_{2}}^{p} D^{\prime}\right)^{-1} J_{E_{2}}^{p}
$$

where $F: E_{1} \rightarrow E_{1}^{*}, K: E_{1}^{*} \rightarrow E_{1}$ are multi-valued and maximal monotone operators, $D: E_{1} \times$ $E_{1}^{*} \rightarrow E_{1}^{*} \times E_{1}$ is defined by $D(x, y)=(F x-y, K y+x)$ for all $(x, y) \in E_{1} \times E_{1}^{*}$, and $F^{\prime}: E_{2} \rightarrow E_{2}^{*}$, $K^{\prime}: E_{2}^{*} \rightarrow E_{2}$ are multi-valued and maximal monotone operators, $D^{\prime}: E_{2} \times E_{2}^{*} \rightarrow E_{2}^{*} \times E_{2}$ is defined by $D^{\prime}(A x, A y)=\left(F^{\prime} A x-A y, K^{\prime} A y+A x\right)$ for all $(A x, A y) \in E_{2} \times E_{2}^{*}$. Then $D$ and $D^{\prime}$ are maximal monotone by Lemma 16 .

When $U=D$ and $T=D^{\prime}$ in Corollary 1, the algorithm (60) becomes

$$
\left\{\begin{array}{l}
g\left(u_{n}, y\right)+\left\langle J_{E_{1}}^{p} C_{n} u_{n}+J_{E_{1}}^{p} G_{n} x_{n}, y-u_{n}\right\rangle+\theta(y)-\theta\left(u_{n}\right) \\
+\frac{1}{r_{n}}\left\langle y-u_{n}, J_{E_{1}}^{p} u_{n}-J_{E_{1}}^{p} x_{n}\right\rangle \geq 0 \forall y \in K \\
y_{n}=J_{E_{1}^{*}}^{q}\left(B_{\delta_{n}}^{D_{n}}\left(J_{E_{1}}^{p} u_{n}-\lambda_{n} A^{*} J_{E_{2}}^{p}\left(I-\Pi_{A K}^{p} B_{\delta_{n}}^{D_{n}^{\prime}}\right) A u_{n}\right)\right) \\
x_{n+1}=J_{E_{1}^{*}}^{q}\left(\eta_{n} J_{E_{1}}^{p}\left(f\left(x_{n}\right)\right)+\left(1-\eta_{n}\right) J_{E_{1}}^{p}\left(S_{n}\left(y_{n}\right)\right)\right) n \geq 1
\end{array}\right.
$$

and its strong convergence is guaranteed, which solves the problem of a common solution of a system of generalized mixed equilibrium problems, split Hammerstein integral equations, and fixed-point problems for the mappings involved in this algorithm.

\section{A Numerical Example}

Let $i=0, E_{1}=E_{2}=\mathbb{R}$, and $K=A K=[0, \infty)$, for $A x=x \forall x \in E_{1}$. The generalized mixed equilibrium problem is formulated as finding a point $x \in K$ such that,

$$
g_{0}(x, y)+\left\langle G_{0} x, y-x\right\rangle+\theta_{0}(y)-\theta_{0}(x) \geq 0, \forall y \in K
$$

Let $r_{0} \in(0,1]$ and define $\theta_{0}=0, g_{0}(x, y)=\frac{y^{2}}{r_{0}}+\frac{2 x^{2}}{r_{0}}$ and $G_{0}(x)=S_{0}(x)=\frac{1}{r_{0}} x$.

Clearly, $g_{0}(x, y)$ satisfies the conditions $(A 1)-(A 4)$ and $G_{0}(x)=S_{0}(x)$ is a Bregman asymptotically non-expansive mapping, as well as a $1-$ inverse strongly monotone mapping. Since $Y_{r_{0}}$ is single-valued, therefore for $y \in K$, we have that

$$
\begin{aligned}
& g_{0}\left(u_{0}, y\right)+\left\langle G_{0} x, y-u_{0}\right\rangle+\frac{1}{r_{0}}\left\langle y-u_{0}, u_{0}-x\right\rangle \geq 0 \\
& \Leftrightarrow \frac{y^{2}}{r_{0}}+\frac{2 u_{0}^{2}}{r_{0}}+\frac{1}{r_{0}}\left\langle y-u_{0}, u_{0}\right\rangle \geq 0 \\
& \Leftrightarrow \frac{y^{2}}{r_{0}}+\frac{2\left|y u_{0}\right|}{r_{0}^{3}}+\frac{x^{2}}{r_{0}} \geq 0 .
\end{aligned}
$$

As (65) is a nonnegative quadratic function with respect to $y$ variable, so it implies that the coefficient of $y^{2}$ is positive and the discriminant $\frac{4 u_{0}^{2}}{r_{0}^{3}}-\frac{4 x^{2}}{r_{0}^{2}} \leq 0$, and therefore $u_{0}=x \sqrt{r_{0}}$. Hence,

$$
\mathrm{Y}_{r_{0}}(x)=x \sqrt{r_{0}} .
$$


By Lemma 13 and (66), $F\left(Y_{r_{0}}\right)=G E P\left(g_{0}, G_{0}\right)=\{0\}$ and $F\left(S_{0}\right)=\{0\}$. Define

$$
\begin{aligned}
& U_{0}, T_{0}: \mathbb{R} \longrightarrow \mathbb{R} \text { by } U_{0}(x)=T_{0}(A x)\left\{\begin{array}{l}
(0,1), x \geq 0 \\
\{1\}, x<0,
\end{array}\right. \\
& P_{[0, \infty)}: \mathbb{R} \longrightarrow[0, \infty) \text { by } P_{[0, \infty)}(A x)=\left\{\begin{array}{l}
0, A x \in(-\infty, 0) \\
A x, A x \in[0, \infty),
\end{array}\right. \\
& B_{\delta}^{U_{0}}=B_{\delta}^{T}: \mathbb{R} \longrightarrow \mathbb{R} \text { by } B_{\delta}^{T}(A y)=B_{\delta}^{U_{0}}(y)=\left\{\begin{array}{l}
\frac{y}{1+(0, \delta)}, y \geq 0 \\
\frac{y}{1+\delta}, y<0,
\end{array}\right. \\
& P_{[0, \infty)} B_{\delta}^{T}: \mathbb{R} \longrightarrow[0, \infty) \text { by } P_{[0, \infty)} B_{\delta}^{T}(A y)=\left\{\begin{array}{l}
\frac{A y}{1+(0, \delta)}, A y \geq 0 \\
0, A y<0 .
\end{array}\right.
\end{aligned}
$$

It is clear that $U_{0}$ and $T_{0}$ are multi-valued maximal monotone mappings, such that $0 \in$ $\operatorname{SOLVIP}\left(U_{0}\right)$ and $0 \in \operatorname{SOLVIP}\left(T_{0}\right)$. We define the $\zeta$-contraction mapping by $f(x)=\frac{x}{2}, \delta_{n}=\frac{1}{2^{n+1}}$, $\eta_{n, 0}=\frac{1}{n+1}, r_{n, 0}=\frac{1}{2^{2 n}}$ and $\zeta=\frac{1}{2}$. Hence, for

$$
\begin{aligned}
& \lambda_{n}=\left\{\begin{array}{l}
\frac{1+\left(0, \frac{1}{2^{n+1}}\right)}{\left|u_{n, 0}\left(1+\left(0, \frac{1}{2^{n+1}}\right)\right)-u_{n, 0}\right|}, u_{n, 0}>0, \\
1, u_{n, 0}=0 \\
\frac{1}{\left|u_{n, 0}\right|}, u_{n, 0}<0
\end{array}\right. \\
& \left\{\begin{array}{l}
u_{n, 0}=\frac{1}{2^{n}} x_{n} \\
y_{n}^{1}=\frac{u_{n, 0}}{1+\left(0, \frac{1}{2^{n+1}}\right)}\left(u_{n, 0}-1\right), u_{n, 0}>0 \\
y_{n}^{2}=\left[\frac{u_{n, 0}}{1+\left(0, \frac{1}{2^{n+1}}\right)}\right]^{2}, u_{n, 0}=0 \\
y_{n}^{3}=\frac{2^{n+1} u_{n, 0}}{2^{n+1}+1}\left(u_{n, 0}+1\right), u_{n, 0}<0 \\
x_{n+1}=\frac{x_{n}}{2(n+1)}+\frac{2^{2 n} n y_{n}}{(n+1)}, n \geq 1
\end{array}\right.
\end{aligned}
$$

we get,

$$
x_{n+1}=\left\{\begin{array}{l}
\frac{x_{n}}{2(n+1)}+\frac{n x_{n}^{2}-2^{n} x_{n}}{(n+1)\left(1+\left(0, \frac{1}{2^{n+1}}\right)\right)}, x_{n}>0 \\
\frac{x_{n}}{2(n+1)}+\frac{n x_{n}^{2}}{(n+1)\left(1+\left(0, \frac{1}{2 n+1}\right)\right)}, x_{n}=0 \\
\frac{x_{n}}{2(n+1)}+\frac{n 2^{n+1}\left(x_{n}^{2}+x_{n}\right)}{2^{n+1}+1}, x_{n}<0 .
\end{array}\right.
$$

In particular,

$$
x_{n+1}=\left\{\begin{array}{l}
\frac{x_{n}}{2(n+1)}+\frac{5\left(n x_{n}^{2}-2^{n} x_{n}\right)}{6(n+1)}, x_{n}>0, \\
\frac{x_{n}}{2(n+1)}+\frac{5 n x_{n}^{2}}{6(n+1}, x_{n}=0, \\
\frac{x_{n}}{2(n+1)}+\frac{n 2^{n+1}\left(x_{n}^{2}+x_{n}\right)}{2^{n+1}+1}, x_{n}<0 .
\end{array}\right.
$$

By Theorem 1 , the sequence $\left\{x_{n}\right\}$ converges strongly to $0 \in \Gamma$. The Figures 1 and 2 below obtained by (MATLAB) software indicate convergence of $\left\{x_{n}\right\}$ given by (32) with $x_{1}=-10.0$ and $x_{1}=10.0$, respectively. 


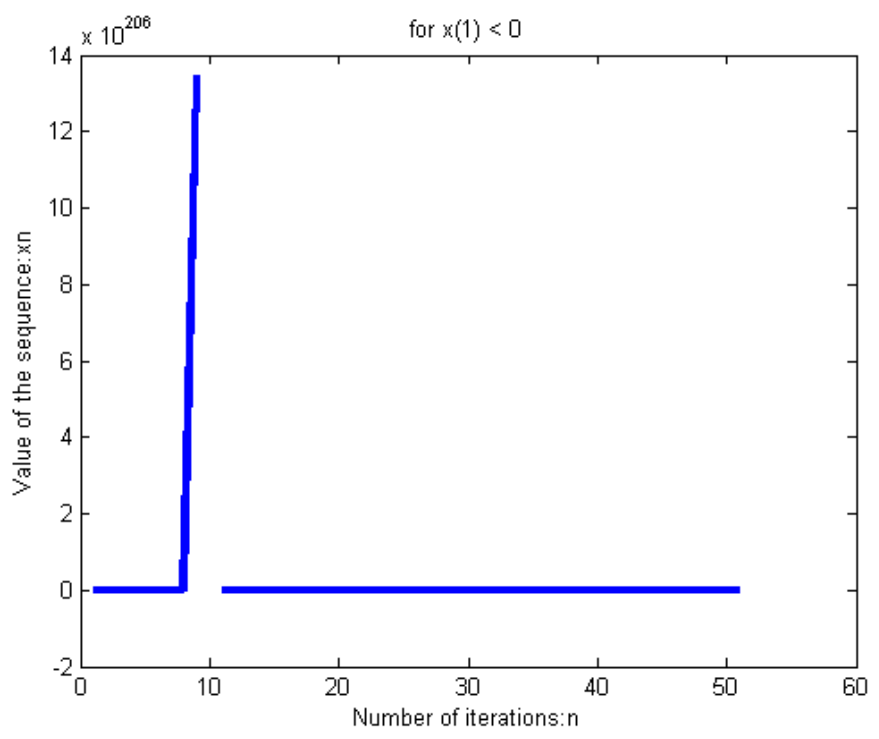

Figure 1. Sequence convergence with initial condition -10.0 .

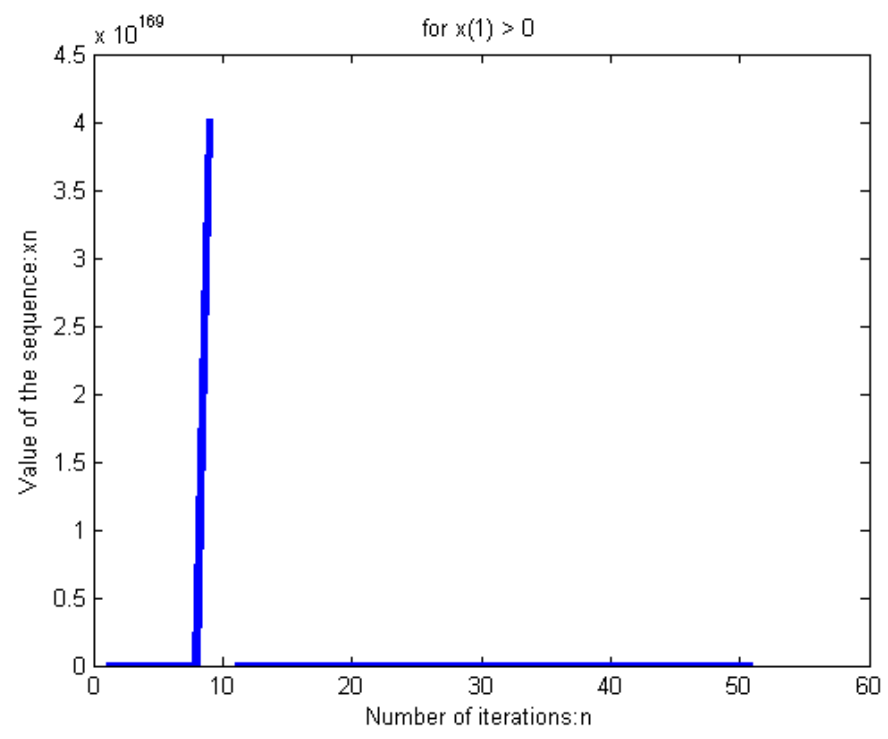

Figure 2. Sequence convergence with initial condition 10.0

Remark 1. Our results generalize and complement the corresponding ones in $[2,7,9,10,22,23]$.

Author Contributions: all the authors contribute equally to all the parts of the manuscript

Funding: This work has been co-funded by the Deanship of Scientific Research (DSR) at University of Petroleum and Minerals (King Fahd University of Petroleum and Minerals KFUPM, Saudi Arabia) through Project No. IN141047 and by the Spanish Government and European Commission through Grant RTI2018-094336-B-I00 (MINECO/FEDER, UE).

Acknowledgments: The author A.R. Khan would like to acknowledge the support provided by the Deanship of Scientific Research (DSR) at University of Petroleum and Minerals (KFUPM)for funding this work through project No. IN141047.

Conflicts of Interest: The authors declare no confilct of interest. 


\section{References}

1. Barbu, V. Maximal Monotone Operators in Banach Spaces, Nonlinear Differential Equations of Monotone Types in Banach Spaces; Springer Monographs in Mathematics; Springer: New York, NY, USA, 2011.

2. Moudafi, A. Split monotone variational inclusions. J. Optim. Theory Appl. 2011, 150, 275-283. [CrossRef]

3. Moudafi, A. Viscosity approximation methods for fixed points problems. J. Math. Anal. Appl. 2000, 241, 46-55. [CrossRef]

4. Kazmi, K.R.; Rizvi, S.H. An iterative method for split variational inclusion problem and fixed point problem for a nonexpansive mapping. Optim. Lett. 2014, 8, 1113-1124. [CrossRef]

5. Nimit, N.; Narin, P. Viscosity Approximation Methods for Split Variational Inclusion and Fixed Point Problems in Hilbert Spaces. In Proceedings of the International Multi-Conference of Engineers and Computer Scientists (IMECS 2014), Hong Kong, China, 12-14 March 2014; Volume II.

6. Schopfer, F.; Schopfer, T.; Louis, A.K. An iterative regularization method for the solution of the split feasibility problem in Banach spaces. Inverse Probl. 2008, 24, 055008. [CrossRef]

7. Chen, J.Z.; Hu, H.Y.; Ceng, L.C. Strong convergence of hybrid Bregman projection algorithm for split feasibility and fixed point problems in Banach spaces. J. Nonlinear Sci. Appl. 2017, 10, 192-204. [CrossRef]

8. Nakajo, K.; Takahashi, W. Strong convergence theorem for nonexpansive mappings and nonexpansive semigroups. J. Math. Anal. Appl. 2003, 279, 372-379. [CrossRef]

9. Takahashi, W. Split feasibility problem in Banach spaces. J. Nonlinear Convex Anal. 2014, 15, 1349-1355.

10. Wang, F.H. A new algorithm for solving multiple-sets split feasibility problem in Banach spaces. Numer. Funct. Anal. Optim. 2014, 35, 99-110. [CrossRef]

11. Payvand, M.A.; Jahedi, S. System of generalized mixed equilibrium problems, variational inequality, and fixed point problems. Fixed Point Theory Appl. 2016, 2016, 93. [CrossRef]

12. Schopfer, F. Iterative Methods for the Solution of the Split Feasibility Problem in Banach Spaces; der Naturwissenschaftlich-Technischen Fakultaten, Universitat des Saarlandes: Saarbrücken, Germany, 2007.

13. Martin, M.V.; Reich, S.; Sabach, S. Right Bregman nonexpansive operators in Banach spaces. Nonlinear Anal. 2012, 75, 5448-5465. [CrossRef]

14. $\mathrm{Xu}, \mathrm{H} . \mathrm{K}$. Existence and convergence for fixed points of mappings of asymptotically nonexpansive type. Nonlinear Anal. 1991, 16, 1139-1146. [CrossRef]

15. Xu, H.K. Inequalities in Banach spaces with applications. Nonlinear Anal. 1991, 16, 1127-1138. [CrossRef]

16. Liu, L.S. Ishikawa and Mann iterative process with errors for nonlinear strongly accretive mappings in Banach spaces. J. Math. Anal. Appl. 1995, 194, 114-125. [CrossRef]

17. Xu, H.K.; Xu, Z.B. An $L_{p}$ inequality and its applications to fixed point theory and approximation theory. Proc. R. Soc. Edinb. 1989, 112A, 343-351. [CrossRef]

18. Aoyamaa, K.; Yasunori, K.; Takahashi, W.; Toyoda, M. Mann Approximation of common fixed points of a countable family of nonexpansive mappings in a Banach space. Nonlinear Anal. 2007, 67, 2350-2360. [CrossRef]

19. Deng, B.C.; Chen, T.; Yin, Y.L. Srong convergence theorems for mixed equilibrium problem and asymptotically I-nonexpansive mapping in Banach spaces. Abstr. Appl. Anal. 2014, 2014, 965737.

20. Reich, S.; Sabach, S. Two strong convergence theorems for Bregman strongly nonexpansive operators in reflexive Banach spaces. Nonlinear Anal. 2010, 73, 122-135. [CrossRef]

21. Chidume, C.E.; Idu, K.O. Approximation of zeros bounded maximal monotone mappings, solutions of Hammertein integral equations and convex minimization problems. Fixed Point Theory Appl. 2016, 2016, 97. [CrossRef]

22. Khan, A.R.; Abbas, M.; Shehu, Y. A general convergence theorem for multiple-set split feasibilty problem in Hilbert spaces. Carpathian J. Math. 2015, 31, 349-357.

23. Ogbuisi, F.U.; Mewomo, O.T. On split generalized mixed equilibrium problem and fixed point problems with no prioir knowledge of operator norm. J. Fixed Point Theory Appl. 2017, 19, 2109-2128. [CrossRef]

(C) 2019 by the authors. Licensee MDPI, Basel, Switzerland. This article is an open access article distributed under the terms and conditions of the Creative Commons Attribution (CC BY) license (http:/ / creativecommons.org/licenses/by/4.0/). 